\title{
ON THE EXTREME EIGENVALUES OF TOEPLITZ OPERATORS OF THE HANKEL TYPE II
}

BY

J. R. DAVIS

1. Introduction. Let $\nu$ be an arbitrary but fixed positive number and set

$$
\mu(x)=x^{2 \nu+1}\left[2^{\nu+1 / 2} \Gamma(\nu+3 / 2)\right]^{-1}, \quad 0 \leqq x<\infty,
$$

and

$$
C_{\nu}=\left[2^{\nu-1 / 2} \Gamma(\nu+1 / 2)\right]^{-1}
$$

We define

$$
J(x)=C_{\nu}^{-1} x^{1 / 2-\nu} J_{\nu-1 / 2}(x), \quad 0 \leqq x<\infty,
$$

where $J_{\nu-1 / 2}$ is the Bessel function of the first kind of order $\nu-1 / 2$. Let $\Lambda \subset[0, \infty)$ be a Borel measurable set and denote by $L_{2, \mu}(\Lambda)$ the Hilbert space of functions defined on $\Lambda$ with inner product $\int_{\Lambda} f g^{*} d_{\mu}$ where $g^{*}$ is the complex conjugate of $g$.

Let $\Omega \subset[0, \infty)$ be a set of positive but finite measure $d_{\mu}$ and $F$ a real bounded function in $L_{1, \mu}([0, \infty))$. We define the operator $B_{A}$ on $L_{2, \mu}(A \Omega)$, $A>0$, by

$$
B_{A} f(x)=\int_{A \Omega} \rho(x, y) f(y) d \mu(y)
$$

where

$$
\rho(x, y)=\int_{0}^{\infty} F(t) J(x t) J(y t) d \mu(t) .
$$

Here $L_{1, \mu}([0, \infty))=L_{1, \mu}$ is the space of all functions defined on $[0, \infty)$ such that $\int|f| d \mu<\infty$.

Under various conditions on $F$ we derive asymptotic formulae for the $k$ th largest eigenvalue of $B_{A}$ as $A \rightarrow \infty$. Our considerations fall into three cases. $F$ will always be a bounded real function in $L_{1, \mu}$ that has a unique maximum at $\xi_{0}, 0 \leqq \xi_{0}<\infty$ and is such that $\lim \sup F(\xi)<F\left(\xi_{0}\right)$ as $\xi \rightarrow \infty$. The three cases are differentiated by the character and position of the maximum and the character of the set $\Omega$. They are

I. $\xi_{0}=0 ; F(\xi) \sim F(0)-\sigma \xi^{\omega}$ as $\xi \rightarrow 0^{+}, \omega>0, \sigma>0 ; \Omega$ as positive and finite measure $d_{\mu}$.

II. $\xi_{0}=0 ; F(\xi) \sim F(0)-L(\xi) \xi^{\omega}$ as $\xi \rightarrow 0^{+}, \omega>0 ; L(\xi)$ is slowly oscil-

Received by the editors March 13, 1964. 
lating as $\xi \rightarrow 0^{+} ; \Omega=|0, a|$.

III. $\xi_{0} \neq 0$ and $F(\xi) \sim F\left(\xi_{0}\right)-\sigma_{1} L\left(\xi-\xi_{0}\right)\left|\xi-\xi_{0}\right|^{\omega}$ as $\xi \rightarrow \xi_{0}^{+}, F(\xi) \sim F\left(\xi_{0}\right)$ - $\sigma_{2} L\left(\xi-\xi_{0}\right)\left|\xi-\xi_{0}\right|^{\omega}$ as $\xi \rightarrow \xi_{0}^{-} ; \omega>0, \sigma_{1}>0, \sigma_{2}>0 ; \Omega=[0, a] ; L(\xi)$ is even and slowly oscillating as $\xi \rightarrow 0$.

As a representative result consider Case I. If $\lambda(A, k)$ is the $k$ th largest eigenvalue of $B_{A}$, then there exists an operator depending only upon $\sigma, \nu$, and $\omega$ such that if $0<\mu(1) \leqq \mu(2) \leqq \cdots$ are its positive eigenvalues

$$
\lambda(A, k)=F(0)-\sigma A^{\cdots \omega}(k)+o\left(A^{-\omega}\right)
$$

as $A \rightarrow \infty$.

In $1953 \mathrm{Kac}$, Murdock and Szegö [4] obtained a result for this problem under rather restrictive conditions in the case of Toeplitz operators of the Fourier type. Parter [5]-[7] and Widom [11]-[14] have studied this case and have weakened the conditions considerably. In |3], I. I. Hirschman reformulated the perturbation theory used by Widom in [14] and obtained results analogous to Widom's for Toeplitz forms associated with Jacobi polynomials. In this paper we follow the format of Hirschman and consider the Toeplitz operators of the Hankel type.

The idea behind the technique is to reformulate the problem so that we can consider a related sequence of operators on a fixed Hilbert space. We then show that this sequence converges suitably to an operator with known eigenvalues and use a perturbation theorem.

The results of this paper constituted part of my doctoral dissertation at Washington University in St. Louis. I wish to express appreciation to Professor I. I. Hirschman for suggesting this problem and to express my thanks for his direction of my career as a graduate student.

2. Preliminaries. In this section we introduce the necessary information concerning the Hankel transform and Bessel function.

If $x, y$, and $z$ are non-negative real numbers, set $J(x, y, x)$ equal to the area of a triangle with sides $x, y$, and $z$ if such exists and zero otherwise. Let

$$
D(x, y, z)=\frac{2^{3 v-52}\lfloor\Gamma(\nu+1 / 2)]^{2}[\Delta(x, y, z)]^{2 \mu-2}}{\Gamma(1 / 2) \Gamma(\nu)(x y z)^{2 \nu-1}} \text {. }
$$

If we define convolution by

$$
f * g \cdot(x)=\int_{0}^{\infty} \int_{0}^{*} f(y) g(z) D(x, y, z) d \mu(y) d \mu(z)
$$

then $L_{1, \mu}$ is a Banach algebra. $D(x, y, z)$ satisfies

$$
\int_{0} D(x, y, z) d \mu(x)=1
$$


The Hankel transform is defined on $L_{1, \mu}$ by

$$
f^{\wedge}(x)=\int_{0}^{\infty} J(x t) f(t) d \mu(t), \quad 0 \leqq x<\infty .
$$

For $f$ and $g$ in $L_{1, \mu}$ we have

$$
(f * g)^{\wedge}=f^{\wedge} g \hat{~}
$$

The Hankel transform on $L_{2, \mu}([0, \infty))=L_{2, \mu}$ is defined by

$$
f^{\wedge}(x)=\int_{0}^{\infty} J(x t) f(t) d \mu(t),
$$

where the partial integrals converge in the norm of $L_{2, \mu}$. This is a unitary mapping of $L_{2, \mu}$ onto $L_{2, \mu}$ and in this case

$$
\left(f^{\wedge}\right)^{\wedge}=f .
$$

These results are all well known and can be found in I. I. Hirschman [2]. We now list some formulas.

$$
\begin{aligned}
|J(x)| & \leqq 1, \\
J(0) & =1, \\
\frac{d}{d z}\left(z^{-\nu} J_{\nu}(z)\right) & =-z^{-\nu} J_{\nu+1}(z), \\
\Delta J(x y) & =-x^{2} J(x y),
\end{aligned}
$$

where

(6)

$$
\begin{gathered}
\Delta=\left(\frac{d}{d y}\right)^{2}+\frac{2 v}{y} \frac{d}{d y} . \\
H_{\nu}^{(1)}(z)=(\pi z / 2)^{-1 / 2} \exp [i(z-\nu \pi / 2-\pi / 4)]
\end{gathered}
$$

$$
\cdot\left[\sum_{m=0}^{M-1}(\nu, m)(-2 i z)^{-m}+O\left(|z|^{-M}\right)\right]
$$

as $|z| \rightarrow \infty$ and $-\pi<\arg z<2 \pi$.

$$
H_{\nu}^{(2)}(z)=(\pi z / 2)^{-1 / 2} \exp [-i(z-\nu \pi / 2-\pi / 4)]
$$

$$
\cdot\left[\sum_{m=0}^{M-1}(\nu, m)(2 i z)^{-m}+O\left(|z|^{-M}\right)\right]
$$

as $|z| \rightarrow \infty$ and $-2 \pi<\arg z<\pi$. 


$$
\begin{array}{r}
J_{\nu}(z)=(\pi z / 2)^{-1 \cdot 2}\{\cos (z-\nu \pi / 2-\pi / 4) \\
\cdot\left[\sum_{m=0}^{m-1}(-1)^{m}(\nu, 2 m)(2 z)^{-2 m-1}+O\left(|z|^{-2 M}\right)\right] \\
\left.-\sin (z-\nu \pi / 2-\pi / 4)\left[\sum_{m=0}^{M-1}(-1)^{m}(\nu, 2 m+1)(2 z)^{-2 m-1}+O\left(|z|^{-2 M-1}\right)\right]\right\}
\end{array}
$$

as $|z| \rightarrow \infty$ and $-\pi<\arg z<\pi$. Here $(\nu, m)$ is Hankel's symbol

$$
(\nu, m)=\Gamma(1 / 2+\nu+m)[m ! \Gamma(1 / 2+\nu-m)]^{-1} .
$$

(2), (3) and (5) can be found in I. I. Hirschman [2], (4) in Watson [10, 47];

(6), (7) and (8) in Erdélyi [1, p. 85].

3. Perturbation theory. Let $0 \leqq S$ be a self-adjoint operator on a separable Hilbert space $\mathscr{E}$ and $F$ a bounded operator on $\mathscr{A}$. We define

$$
\mathscr{S}=\left\{f \mid F f \in \mathscr{D}\left(S^{1 / 2}\right)\right\} .
$$

Here $S^{1 / 2}$ is the unique positive square root of $S$ and $\mathscr{D}\left(S^{1_{1} 2}\right)$ is the domain of $S^{1 / 2}$. Let $\mathscr{M}$ be the closure of $\mathscr{S}$ in $\mathscr{H}$. Then $\mathscr{M}$ is a closed subspace of $\mathscr{H}$ and is itself a Hilbert space.

THEOREM 3a. With the above definitions, there exists a self-adjoint operator $S_{F}$ on the Hilbert space $\mathscr{M}$ with the properties
(1) $\mathscr{D}\left(S_{F}\right) \subseteq \mathscr{S}$,
(2) $\left(S_{F} f \mid g\right)=\left(S^{1 / 2} F f \mid S^{1 / 2} F g\right)$ for all $f \in \mathscr{D}\left(S_{F}\right)$ and $g \in \mathscr{Y}$.

Proof. See F. Riesz and B. Sz.-Nagy [8, p. 326].

Let $A$ be a closed linear operator on $\mathscr{E}$, not necessarily densely defined.

Definition $3 b$. A subset $\mathscr{C}$ of $\mathscr{D}(A)$ is said to be a core for $A$ if $\{(f, g) \mid g$ $=A f, f \in \mathscr{L}\} \subset \mathscr{H} \times \mathscr{W}$ is dense in $\{(f, g) \mid g=A f, f \in \mathscr{D}(A)\}$; that is, if $\mathscr{C} \times A \mathscr{C}$ is dense in the graph of $A$.

Definition 3c. Let $A_{n}, n=1,2, \cdots$, and $A$ be closed linear operators in $\mathscr{H}$ and let $\mathscr{E}=\left\{f \mid A_{n} f \rightarrow A f\right.$ as $\left.n \rightarrow \infty\right\}$. If $\mathscr{C}=\mathscr{D}(A)$ we say that $A$ is the strong limit of the $A_{n}$ 's. If $\mathscr{E}$ is a core for $A$ we say that $A$ is the closure of the strong limit of the $A_{n}$ 's.

We will use " $\rightarrow$ " for strong convergence in a Hilbert space and " - " for weak convergence.

Theorem 3d. Suppose the following conditions are satisfied:

(i) $0 \leqq S$ is a self-adjoint operator in $\mathbb{Z}$;

(ii) $F$ is a bounded operator in $\mathscr{4}$;

(iii) $0 \leqq S_{n}$ is a self-adjoint operator in $\mathscr{A}, n=1,2,3, \cdots, F_{n}$ is a bounded operator in $\mathscr{H}, n=1,2,3, \cdots$ such that $\mathscr{K}\left(F_{n}\right) \subset \mathscr{Y}\left(S_{n}\right), n=1,2, \cdots$; 
(iv) $F$ is the strong limit of $F_{n}$ as $n \rightarrow \infty$;

(v) $S^{1 / 2}$ is the closure of the strong limit of $S_{n}^{1 / 2}$ as $n \rightarrow \infty$;

(vi) $S^{1 / 2} F$ is the closure of the strong limit of $S_{n}^{1 / 2} F_{n}$ as $n \rightarrow \infty$.

Set $S_{n, F}=F_{n}^{*} S_{n} F_{n}$, where $F_{n}^{*}$ is the adjoint of $F_{n}$ and let $S_{n, F}=\int_{0}^{\infty} \lambda d \psi_{n}(\lambda)$ be the spectral resolution of $S_{n, F}$ on $\mathscr{H}$. Here we assume $\psi_{n}(\lambda)=\psi_{n}\left(\lambda^{+}\right)$for $0 \leqq \lambda<\infty$ and $n=1,2, \cdots$ and that $\psi_{n}\left(0^{-}\right)=0$.

Let

$$
S_{F}=\int_{0}^{\infty} \lambda d \psi(\lambda)
$$

be spectral resolution of $S_{F}$ on $\mathscr{M}$ and again we assume that $\psi(\lambda)=\psi\left(\lambda^{+}\right)$for $0 \leqq \lambda<\infty$ and $\psi\left(0^{-}\right)=0$. Then for every $f \in \mathbb{E}$ and $\lambda$ not in the point spectrum of $S_{F}$

$$
\psi_{n}(\lambda) f \rightarrow \psi(\lambda) f
$$

where " $\rightarrow$ " is in $\mathscr{\&}$.

Proof. See I. I. Hirschman [3].

We note that if $\mathscr{H}_{1}$ is a subspace of $\mathscr{H}$ and if $E$ is a projection in $\mathscr{H}_{1}$ considered as a Hilbert space, then $E$ can be considered as a projection in $\mathscr{W}$, namely, as the projection of $\mathscr{E}$ on $E \mathscr{H}_{1}$. It is in this sense that the above convergence is in $\mathscr{H}$. This convention will be used throughout.

Theorem 3e. Suppose that $0 \leqq R_{n}, n=1,2,3, \cdots$ are bounded self-adjoint operators defined on subspaces $\mathscr{N}_{n}$ of a Hilbert space $\mathscr{H}$. Let $0<R$ be a selfadjoint operator defined on a subspace $\mathscr{N}$ of $\mathscr{H}$. Let

$$
\begin{gathered}
R_{n}=\int_{0}^{\infty} \lambda d E_{n}(\lambda), \\
R=\int_{0}^{\infty} \lambda d E(\lambda)
\end{gathered}
$$

be the spectral resolutions of $R_{n}$ on $\mathscr{N}_{n}$ and of $R$ on $\mathscr{N}$.

Suppose further that:

(a) $E_{n}(\lambda) \rightarrow E(\lambda)$ as $n \rightarrow \infty$ for all $\lambda>0$ and not in the point spectrum of $R$. Here " $\rightarrow$ " is in $\mathbb{E}$;

(b) there is a number $m>0$ such that if $f_{n} \in \mathscr{N}_{n},\left\|f_{n}\right\|=1$ and $\left(R_{n} f_{n} \mid f_{n}\right)$ $\leqq m_{1}<m$ for $n \in p_{1}$ then $p_{1}$ contains a subsequence $p_{2}$, where $f_{n} \rightarrow f$ as $n \rightarrow \infty$ in $p_{2}$ and $f \neq 0$. Here " $\rightarrow$ " is in $\mathscr{E}$ and $p_{k}, k=1,2$, denote subsequences of the natural numbers.

Then

$$
\begin{gathered}
\operatorname{dim} E(\lambda)<\infty, \quad 0 \leqq \lambda<m, \\
\operatorname{dim} E_{n}(\lambda) \rightarrow \operatorname{dim} E(\lambda) \quad \text { as } n \rightarrow \infty \quad \text { for } 0 \leqq \lambda<m
\end{gathered}
$$

and $\lambda$ not in the point spectrum of $R$. 
Proof. See I. I. Hirschman [3].

In order to apply Theorem $3 \mathrm{e}$ we will need the following result.

Theorem $3 \mathrm{f}$. $F \mathscr{M}$ reduces $S_{F}$.

Proof. Let $f \in F \mathscr{S}$. Clearly, if $g \in \mathscr{S}$, then $F g \in \mathscr{S}$. Then writing $g=F g$ $+(I-F) g$ we see that $(I-F) g \in \mathscr{S}$. Thus $(I-F) \mathscr{S} \subset \mathscr{S}$ and as $\mathscr{S}$ is dense in $\mathscr{M},(I-F) \mathscr{S}$ is dense in $(I-F) \mathscr{M}$. Also $F \mathscr{S}$ is dense in $F \mathscr{M}$. Let $h \in(I-F) \mathscr{S}$. Then by (2) we have

$$
\left(S_{F} f \mid h\right)=\left(S^{1 / 2} F f \mid S^{1 / 2} F h\right)=0
$$

and hence $S_{F} f \in F \mathscr{M}$.

4. Definitions and preliminaries-Case I. We shall assume that $F(\xi)$ satisfies the following conditions:

(i) $F(\xi)$ is a bounded real function in $L_{1, \mu}$.

(ii) $F(0)=M$ is the unique maximum of $F$ and $\lim \sup F(\xi)<M$ as $\xi \rightarrow \infty$.

(iii) $F(\xi) \sim M-\sigma \xi^{\omega}$ as $\xi \rightarrow 0^{+}, \omega>0, \sigma>0$.

We also assume that $\Omega \subseteq[0, \infty)$ has positive but finite measure $d \mu$.

We will set up an apparatus, part of which may seem superfluous for this case, but which sets a pattern for the later cases where it will be necessary.

We define four Hilbert spaces $\mathscr{H}, \mathscr{H}^{i}, \mathscr{C}^{i}, \mathscr{C}$, all equal to $L_{2, \mu}$. We will use the variable $x$ for functions in $\mathscr{H}, \xi$ for $\mathscr{H}^{n}, t$ for $\mathscr{C}^{\wedge}$, and $y$ for $\mathscr{C}$. This will be the convention throughout this paper. For Case I and Case II a function denoted by $f^{-}$will always be in $\mathscr{H}^{-}$or $\mathscr{L}^{-}$and will always be the Hankel transform of a function in $\mathscr{E}$ or $\mathscr{C}$, respectively.

We define unitary maps $\phi$ of $\mathscr{H}$ onto $\mathscr{H}^{2}$ and $\psi$ of $\mathscr{C}$ onto $\mathscr{L}^{-}$by

$$
\phi f \cdot(\xi)=f^{\wedge}(\xi)
$$

and

$$
\begin{gathered}
\psi f \cdot(t)=f^{\wedge}(t) . \\
\text { We define maps } \chi_{A} \text { of } \mathscr{H}^{-} \text {onto } \mathscr{C}^{-} \text {and } \chi_{A}^{*} \text { of } \mathscr{C}^{-} \text {onto } \mathscr{H}^{-} \text {by } \\
\chi_{A} f \cdot(t)=f\left(t A^{-1}\right) A^{-\nu-1 / 2}
\end{gathered}
$$

and

$$
\chi_{A}^{*} f \cdot(\xi)=f(A \xi) A^{\nu+1 / 2} .
$$

It is evident that $\chi_{A}, \chi_{A}^{*}$ are unitary and that $\chi_{A}^{*}$ is the adjoint of $\chi_{A}$.

Define the projection $E_{A}$ in $\mathscr{E}$ by

$$
E_{A} f(x)=C_{A \Omega}(x) f(x),
$$

where $C_{A \Omega}$ is the characteristic function of $A \Omega$.

$E_{A}$ is unitarily equivalent to the projection $E_{A}^{\hat{A}}$ in $\mathscr{H}^{-}$and $F_{A}^{\hat{A}}$ in $\mathscr{C}^{-} \mathrm{de}-$ fined by 


$$
\begin{aligned}
& E_{A}^{\hat{A}}=\phi E_{A} \phi^{-1}, \\
& F_{A}^{\hat{A}}=\chi_{A} E_{A} \chi_{A}^{*} .
\end{aligned}
$$

We define the operator $T^{\wedge}$ in $\mathscr{H}^{\wedge}$ by

$$
T^{\wedge} f \cdot(\xi)=(M-F(\xi)) f(\xi) .
$$

$T^{\wedge}$ is unitarily equivalent to the operators $T$ in $\mathscr{H}$ and $T_{A}^{\wedge}$ in $\mathscr{C}^{-}$defined by

$$
\begin{aligned}
T & =\phi^{-1} T^{\wedge} \phi, \\
T_{A}^{-} & =\chi_{A} T^{-} \chi_{A}^{*} .
\end{aligned}
$$

We define the projection $F$ in $\mathscr{C}$ by

$$
F f \cdot(y)=C_{\Omega}(y) f(y) .
$$

$F$ is unitarily equivalent to the projection $F^{-}$in $\mathscr{C}^{-}$defined by

$$
F^{\wedge}=\psi F \psi^{-1} \text {. }
$$

Finally we define the operators $S_{\hat{A}}$ and $S^{\wedge}$ on $\mathscr{C}^{\wedge}$ by

$$
\begin{aligned}
& S_{A}^{\wedge} f \cdot(t)=\sigma^{-1} A^{\omega} T_{A}^{\wedge} f \cdot(t), \\
& S^{\wedge} f \cdot(t)=\sigma^{-1} t^{\omega} f(t) .
\end{aligned}
$$

If $\lambda(A, 1) \geqq \lambda(A, 2) \geqq \cdots$ are the positive eigenvalues of $B_{A}$ then $M$ $-\lambda(A, 1) \leqq M-\lambda(A, 2) \leqq \cdots$ are the eigenvalues of

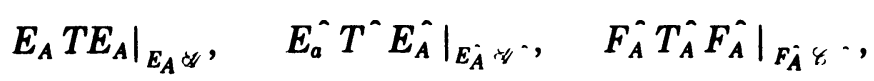

where these symbols are to read " $E_{A} T E_{A}$ restricted to $E_{A} \mathscr{K}$," etc. The eigenvalues of

$$
\left.F_{A}^{\wedge} S_{A}^{\wedge} F_{A}\right|_{F_{A} \hat{\gamma}^{\circ}}
$$

are $(M-\lambda(A, 1)) A^{\omega} \sigma^{-1} \leqq(M-\lambda(A, 2)) A^{\omega} \sigma^{-1} \leqq \ldots$ In the following sections we will show that $F_{A} \hat{S_{A}} F_{A}^{\hat{n}}$ converges to $S_{F^{-}}$of Theorem $3 \mathrm{a}$ as $A \rightarrow \infty$ so that if $0<\mu(1) \leqq \mu(2) \leqq \cdots, \lim \mu(k)=\infty$, are the positive eigenvalues of $\left.S_{F}\right|_{F}$, then using the perturbation theorem we will have

$$
(M-\lambda(A, k)) \sigma^{-1} A^{\omega}=\mu(k)+o(1)
$$

as $A \rightarrow \infty$ for $k=1,2, \cdots$, or equivalently

$$
\lambda(A, k)=M-\mu(k) A^{-\omega} \sigma+o\left(A^{-\omega}\right) .
$$

Recall that $S_{F^{-}}$is a self-adjoint operator defined on a subspace $\mathscr{M}^{-}$of $\mathscr{C}^{-}$ and $\mathscr{D}\left(S_{F^{-}}\right) \subseteq \mathscr{S}^{-}$, where

$$
\mathscr{S}^{\wedge}=\left\{f \mid F^{\wedge} f \in \mathscr{D}\left(\left(S^{\wedge}\right)^{1 / 2}\right)\right\}=\mathscr{D}\left(\left(S^{\wedge}\right)^{1 / 2} F^{\wedge}\right) .
$$

Recall also that by Theorem $3 \mathrm{f}, F^{\wedge} \mathscr{M}^{-}$reduces $\hat{S^{-}}$so that $\left.S_{F^{-}}\right|_{F^{-}}$is 
an operator in $F^{\wedge} \mathscr{M}^{\wedge}$. Since this is the operator that we will eventually be interested in it is important that we determine the nature of $F^{\wedge} \mathscr{M}^{\prime}$.

It is clear that if $\Omega$ is an interval then $\mathscr{Y}^{\wedge}$ is dense in $\mathscr{C}^{\wedge}$ and thus $F^{\wedge} \mathscr{M}^{-}$ $=F^{\wedge} \mathscr{C}^{\wedge}$. Suppose that $\Omega$ is a nowhere dense set of positive measure and that $\omega>1$. Let $f^{\wedge} \in F^{\wedge} \mathcal{Y}^{\wedge}$. Then $t^{\mu / 2} f^{\wedge} \in L_{2, \mu}$ and $f$ has its support on $\Omega$. We claim that $f$ is continuous. Indeed, for $0<y_{0}<\infty$ and $\epsilon>0$ such that $1+\epsilon=\omega$,

$$
\begin{aligned}
\mid f(y) & -\left.f\left(y_{0}\right)\right|^{2} \\
& \leqq \int_{0}^{\infty}\left|f^{-}(t)\right|^{2} t^{1+\iota} d \mu(t) \int_{0}^{\infty}\left|J_{\nu-1 / 2}(y t) y^{1 / 2-\nu}-J_{\nu-1 / 2}\left(y_{0} t\right) y_{0}^{1 / 2-\nu}\right|^{2} C_{\nu}^{-1} t^{-\iota} d t .
\end{aligned}
$$

Since $J_{\nu-1 / 2}(t)=O\left(t^{-1 / 2}\right)$ as $t \rightarrow \infty$, see (7) §2,

$$
\int_{0}^{\infty}\left|J_{\nu-1 / 2}(y t) y^{1 / 2-\nu}-J_{\nu-1 / 2}\left(y_{0} t\right) y_{0}^{1 / 2-\nu}\right|^{2} t^{-\epsilon} d t \rightarrow 0
$$

as $y \rightarrow y_{0}$.

But $f$ vanishes on the complement of $\Omega$, an everywhere dense set, and hence vanishes indentically. Thus we see that $F^{\wedge} \mathscr{S}^{\text {n }}$ contains only the zero function.

At first it might seem possible that given an integer $n$ an $\Omega$ could be found such that the dimension of $F^{\wedge} \mathscr{M}^{\wedge}$ would be $n$. This is not the case. We will show that if $F^{\wedge} \mathscr{M}^{\wedge}$ contains a function other than the zero function, its dimension is infinite.

Lemma 4a. Let $W(x)$ be positive, nondecreasing and such that $W(x+y)$ $\leqq W(x) W(y)$. Let $f(y)$ be such that $\int_{0}^{\infty}|f(y)|^{2} W(x) d \mu(x)<\infty$ and let $g(y)$ be such that $\int_{0}^{\infty}|g(y)| W(x)^{1 / 2} d \mu(x)<\infty$. Then

$$
\int_{0}^{\infty}|f * g \cdot(y)|^{2} W(y) d \mu(y)<\infty .
$$

Proof. We have, using Schwarz's inequality,

$$
|f * g \cdot(y)|^{2} \leqq C_{1} \int_{0}^{\infty} \int_{0}^{\infty}|f(z)|^{2}|g(x)| W(x)^{-1 / 2} D(x, y, z) d \mu(z) d \mu(x),
$$

where

$$
C_{1}=\int_{0}^{\infty}|g(x)| W(x)^{1 / 2} d \mu(x) .
$$

Since $D(x, y, z)=0$ for $y \geqq x+z$ we may assume $y<x+z$ and hence $W(y)$ $\leqq W(x+z) \leqq W(x) W(z)$. Thus, using (1) §2 we get 


$$
\begin{aligned}
\int_{0}^{\infty}|f * g \cdot(y)|^{2} W(y) d \mu(y) \\
\quad \leqq C_{1} \int_{0}^{\infty} \int_{0}^{\infty} \int_{0}^{\infty}|f(z)|^{2} W(z)|g(x)| W(x)^{1 / 2} D(x, y, z) d_{\mu}(z) d_{\mu}(x) d_{\mu}(y) \\
\quad \leqq C_{1}^{2} \int_{0}^{\infty}|f(z)|^{2} W(z) d_{\mu}(z)<\infty .
\end{aligned}
$$

Now suppose there exists an $f^{\wedge} \in F^{\wedge} \mathscr{M}^{\wedge}, f^{\wedge} \neq 0$. Let $\Omega_{1} \subseteq \Omega$ be the support of $f$ and let $I$ be an interval such that the $d_{\mu}$ measure of $I \cap \Omega_{1}$ is positive. Let $h(y)$ be an infinitely differentiable, positive function that vanishes off $I$ and set $W(t)=(1+t)^{\omega}$. Since $F(f h)=f h$ we have $f^{\wedge} * h^{\wedge} \in F^{\wedge} \mathscr{C}^{\wedge}$ and applying Lemma 4 a we get that $f^{-} * h^{\wedge} \in F^{-} \mathscr{M}^{-}$.

Let $I_{n}, n=1,2, \cdots$, be a sequence of disjoint intervals such that the $d_{\mu}$ measure of $I_{n} \cap \Omega_{1}$ is positive. The corresponding sequence of functions $f^{\wedge} * h_{n}, n=1,2, \cdots$ will be independent and hence $F^{\wedge} \mathscr{M}^{-}$has infinite dimension.

In general $F^{\wedge} \mathscr{M}^{\wedge} \neq F^{\wedge} \mathscr{C}^{\wedge}$. For example, let $\Omega$ be the disjoint union of a nowhere dense set of positive measure and a finite interval.

If $F^{\wedge} \mathscr{M}^{\wedge}=\{0\}, S_{F^{-}}$has no eigenvalues, but by convention we will say it has infinitely many all equal to plus infinity.

5. Convergence of operators-Case $I$.

Lemma 5a. Suppose $L, L_{n}$ are multiplier transformations on $\mathscr{C}$

$$
\begin{gathered}
L u \cdot(t)=u(t) h(t), \\
L_{n} u \cdot(t)=u(t) h_{n}(t) .
\end{gathered}
$$

Then if

$$
\lim _{n \rightarrow \infty} h_{n}(t)=h(t)
$$

$$
\left|h_{n}(t)\right| \leqq K|h(t)|
$$

$L$ is the strong limit of the $L_{n}$.

Proof. Routine.

TheOREM $5 \mathrm{~b} .\left(S^{\wedge}\right)^{1 / 2}$ is the strong limit of $\left(S_{\hat{A}}\right)^{1 / 2}$.

Proof. We have

$$
\widehat{S_{A}} \hat{f} \cdot(t)=s_{A}(t) f(t),
$$

where

$$
s_{A}(t)=\sigma^{-1} A^{\omega}\left(M-F\left(t A^{-1}\right)\right) .
$$


By condition (iii) on $F$

$$
s_{A}(t) \rightarrow t^{\omega} \quad \text { as } A \rightarrow \infty .
$$

Now $M-F(\xi)=\sigma \xi^{\omega} \epsilon(\xi)$ for $0 \leqq \xi \leqq 1$, where $\epsilon(\xi)$ is bounded and $\epsilon(\xi) \rightarrow 1$ as $\xi \rightarrow 0^{+}$.

Using this for $0 \leqq t \leqq A$ and the fact that $F$ is bounded for $t>A$, we obtain

$$
s_{A}(t) \leqq C t^{\omega}
$$

for all $A$ and $t$. (1) and (2) are precisely the conditions for Lemma $5 \mathrm{a}$ and the theorem is proved.

We now compute $\hat{F_{A}}$. From the definition

$$
\hat{F_{A}}=\chi_{A} \phi E_{A} \phi^{-1} \chi_{A}^{*} \text {. }
$$

A straightforward computation shows that $F_{\hat{A}}=F^{\wedge}$ for all $A$. Since $\left(S_{\hat{A}}^{\hat{A}}\right)^{1 / 2}$ converges strongly to $\left(S^{\wedge}\right)^{1 / 2}$, it is immediate that $\left(S^{\wedge}\right)^{1 / 2} F^{-}$is the strong limit of $\left(\hat{S_{A}}\right)^{1 / 2} F^{-}$.

6. The asymptotic formula-Case $I$. Let $S_{F^{-}}^{-}$be constructed from $F^{-}$and $S^{\wedge}$ as in Theorem 3 a.

Then

$$
\mathscr{D}\left(\hat{S}_{F^{-}}\right) \subseteq \mathscr{S}^{\wedge}=\left\{f \mid F^{\wedge} f \in \mathscr{D}\left(\left(S^{\wedge}\right)^{1 / 2}\right)\right\}
$$

and $\hat{S_{F^{-}}}$is a self-adjoint operator on $\mathscr{M}^{-}=$closure of $\mathscr{S}^{\circ}$.

Let

$$
\hat{S^{-}}=\int_{0}^{\infty} \lambda d \psi^{\hat{\prime}}(\lambda)
$$

be the spectral resolution of $S_{F^{-}}$on $\mathscr{M}^{\wedge}$ and let

$$
\hat{S_{A, F^{-}}}=\int_{0}^{\infty} \lambda d \psi_{\hat{A}}(\lambda)
$$

be the spectral resolution of $F^{\wedge} S_{A}^{\hat{A}} F^{\wedge}=\hat{S_{A, F^{\wedge}}}$. By Theorem 3 d, we have

$$
\hat{\psi_{A}}(\lambda) \rightarrow \psi^{\hat{*}}(\lambda)
$$

for $0 \leqq \lambda<\infty$, and $\lambda$ not in the point spectrum of $S_{F^{*}}$.

Let $R^{-}$be $S_{F^{-}}^{-}$restricted to $F^{-} \mathscr{M}^{-}$and $R_{A}^{\wedge}$ be $S_{A, F^{-}}$restricted to $F^{\wedge} \mathscr{C}^{-}$. It is easy to show that $R^{\wedge}>0$ and $R_{A}^{\wedge}>0$. Then $R^{\wedge}$ has the spectral resolution on $F^{\wedge} \mathscr{M}^{\wedge}$,

$$
R^{\wedge}=\int_{0}^{\infty} \lambda d G^{\wedge}(\lambda)
$$

where $G^{\wedge}(\lambda)=\psi^{\wedge}(\lambda)-\psi^{\wedge}(0)$. For $0 \leqq \lambda<\infty$ and $G^{\wedge}(0)=0$, and $R_{\hat{A}}^{\wedge}$ has the spectral resolution on $F^{\wedge} \mathscr{C}^{\prime}$, 


$$
R_{\hat{A}}=\int_{0}^{\infty} \lambda d G_{\hat{A}}(\lambda)
$$

where $G_{A}^{\hat{A}}(\lambda)=\psi_{\hat{A}}(\lambda)-\psi_{\hat{A}}(0)$ for $0 \leqq \lambda<\infty$ and $G_{\hat{A}}^{\hat{(}}(0)=0$. Since $\psi^{\wedge}(0)$ $=I-F^{\wedge}$ and $\psi_{A}(0)=I-F^{\wedge}$, where $I$ is the identity operator, it follows from (1) that

$$
G_{\hat{A}}^{\hat{n}}(\lambda) \rightarrow G^{\wedge}(\lambda)
$$

for all $\lambda, 0 \leqq \lambda<\infty, \lambda$ not in the point spectrum of $R^{\wedge}$.

Lemma 6a. If $f_{n}^{\wedge} \in F^{\wedge} \mathscr{C}^{\wedge},\left\|f_{n}^{\wedge}\right\|=1, n=1,2,3, \cdots$, and $f_{n}^{\wedge} \rightarrow f^{\wedge}$ as $n \rightarrow \infty$, then $f_{n}^{\wedge}(t) \rightarrow f^{\wedge}(t)$ uniformly on any compact set $Z$.

Proof. $f_{n}^{\wedge} \in F^{\wedge} \mathscr{C}^{\wedge}$ implies $F^{\wedge} f_{n}^{\wedge}=f_{n}^{\wedge}$; that is,

$$
\hat{f}_{n}^{\hat{n}}(t)=\int_{0}^{\infty} f_{n}(y)\left[J(y t) C_{\Omega}(y)\right] d \mu(y) \text {. }
$$

Now $f_{n}^{\wedge} \rightarrow f^{\wedge}$ implies $f_{n} \rightarrow f$ and since $J(y t) C_{\Omega}(y) \in L_{2, \mu}$ we have $f_{n}^{\wedge}(t) \rightarrow f^{\wedge}(t)$ for each fixed $t$ as $n \rightarrow \infty$.

Now

$$
\hat{f_{n}^{\wedge}}(t)-\hat{f_{n}}(s)=\int_{0}^{\infty} f_{n}(y)[J(y t)-J(y s)] C_{\Omega}(y) d \mu(y)
$$

and by Schwarz's inequality, we get

$$
\left|f_{n}^{\hat{n}}(t)-f_{n}^{\hat{n}}(s)\right| \leqq \int_{0}^{\infty}[J(y t)-J(y s)]^{2} C_{\Omega}(y) d \mu(y) .
$$

This implies that $\left\{f_{n}^{\hat{n}}(t)\right\}$ is an equicontinuous set of functions and hence $f_{n}^{\wedge}(t) \rightarrow f^{\wedge}(t)$ uniformly on any compact set.

We denote by $p$ a subsequence of the natural numbers and let $A(1)<A(2)$ $<\cdots$, where $A(k) \rightarrow \infty$ as $k \rightarrow \infty$.

Lemma 6b. With the above definitions let $f_{n} \in F^{\wedge} \mathscr{C}^{\wedge},\left\|f_{n}\right\|=1,\left(R_{\hat{A}(n)} f_{n} \mid f_{n}\right)$ $\leqq m<\infty$ for $n \in \boldsymbol{p}$. If $f_{n} \rightarrow f$ as $n \rightarrow \infty$ in $\boldsymbol{p}_{1}, \boldsymbol{p}_{1}$ a subsequence of $\boldsymbol{p}$, then $f \neq 0$.

Proof. We claim that given $m_{1}>0$ there exists numbers $t_{0}$ and $A_{0}$ such that $s_{A}(t)>m_{1}$ for $t>t_{0}$ and $A>A_{0}$. Since $M-F(\xi)=\sigma \xi^{\omega} \epsilon(\xi)$ for $0 \leqq \xi \leqq 1$, where $0<\epsilon(\xi) \rightarrow 1$ as $\xi \rightarrow 0^{+}$, we have for $0 \leqq t A^{-1} \leqq 1$ that $\epsilon\left(t A^{-1}\right)>m_{2}$ $>0$ and $s_{A}(t)=t^{\omega} \epsilon\left(t A^{-1}\right)>m_{2} t^{\omega}$. For $t A^{-1}>1, \sigma^{-1}\left[M-F\left(t A^{-1}\right)\right]>m_{3}$ $>0$ and $s_{A}(t)=\sigma^{-1} A^{\omega}\left(M-F\left(t A^{-1}\right)\right)>m_{3} A^{\omega}$, etc.

Now pick $n_{0}$ and $t_{0}$ so that $s_{A(n)}(t)>2 m$ for $n>n_{0}, t>t_{0}$. Then for $n>n_{0}$

$$
m \geqq\left(R_{\hat{A(n)}} f_{n} \mid f_{n}\right) \geqq 2 m \int_{t_{0}}^{\infty}\left|f_{n}(t)\right|^{2} d \mu(t)
$$

and hence 


$$
\int_{0}^{t_{0}}\left|f_{n}(t)\right|^{2} d_{\mu}(t) \geqq 1 / 2 \text {. }
$$

But by Lemma $6 a, f_{n}(t) \rightarrow f(t)$ uniformly on $\left[0, t_{0}\right]$ and hence $\|f\|^{2} \geqq 1 / 2$.

Theorem 6c. Let $F(\xi)$ satisfy conditions (i), (ii), (iii), of $\$ 4$ and let $\Omega$ be $a$ set of positive but finite measure $d_{\mu}$. Let $\lambda(A, 1) \geqq \lambda(A, 2) \geqq \cdots$ be the positive eigenvalues of $B_{A}$ and $0<\mu(1) \leqq \mu(2) \leqq \cdots, \mu(k) \rightarrow \infty$ as $k \rightarrow \infty$, be the positive eigenvalues of $S_{F^{-}}$, where $\mu(k)=+\infty, k=1,2,3, \cdots$ if $F^{\wedge} \mathscr{M}^{\wedge}=\{0\}$.

Then

$$
\lambda(A, k)=M-\sigma A^{-\omega} \mu(k)+o\left(A^{-\omega}\right) .
$$

Proof. Lemma $6 \mathrm{~b}$ and (2) are the hypotheses for Theorem $3 \mathrm{e}$ and hence

$$
\sigma^{-1} A^{\omega}(n)(M-\lambda(A(n), k))=\mu(k)+o(1) .
$$

But this is equivalent to (3).

7. Definitions and preliminaries-Case II. We say that a function $L$ is slowly oscillating as $\xi \rightarrow 0^{+}$if for all $\epsilon>0$

$$
\begin{gathered}
\xi^{\epsilon} L(\xi) \text { is increasing in a neighborhood of } 0, \xi>0, \\
\xi^{-\epsilon} L(\xi) \text { is decreasing in a neighborhood of } 0, \xi>0 \text {. }
\end{gathered}
$$

We shall assume that $F$ satisfies the following conditions:

(i) $F$ is a bounded real-valued function in $L_{1, \mu}$.

(ii) $F$ has a unique maximum $M$ at $\xi=0$ and $\lim \sup F(\xi)<M$ as $\xi \rightarrow \infty$.

(iii) $M-F(\xi) \sim \xi^{\omega} L(\xi)$ as $\xi \rightarrow 0^{+}$, where $L(\xi)$ is positive, continuous on $0<\xi<\infty, L(\xi)=O(1)$ as $\xi \rightarrow \infty$, bounded away from 0 as $\xi \rightarrow \infty$ and is slowly oscillating as $\xi \rightarrow 0^{+}$.

Define the Hilbert spaces $\mathscr{H}, \mathscr{C}^{\wedge}, \mathscr{C}$, and $\mathscr{C}^{\circ}$ and the operators $\phi, \psi, \chi_{A}$, $\chi_{A}^{*}, E_{A}, E_{A}^{\hat{A}}, F_{A}^{\wedge}, T^{\wedge}, T_{A}^{\hat{A}}, F$ and $F^{\wedge}$ as in $\$ 4$.

In this case we assume that $\Omega=[0, a]$ and without loss of generality that $a=1$.

The operators $S_{A}$ and $S^{\wedge}$ on $\mathscr{C}^{\wedge}$ are different than in $\$ 4$ and are defined by

$$
\begin{aligned}
& \hat{S_{A}} f \cdot(t)=A^{\omega}\left(L\left(A^{-1}\right)\right)^{-1} T_{\hat{A}}^{\hat{f}} f \cdot(t), \\
& S \hat{f} \cdot(t)=t^{\omega} f(t) \text {. }
\end{aligned}
$$

As in Case I, if $\lambda(A, 1) \geqq \lambda(A, 2) \geqq \cdots$ are the positive eigenvalues of $B_{A}$ then the eigenvalues of $F_{A} \hat{S_{A}} F_{A}$ restricted to $F_{A}^{\hat{C} \mathscr{C}^{\wedge}}$ are $(M-\lambda(A, 1)) A^{\omega}$ $\left[L\left(A^{-1}\right)\right]^{-1} \leqq(M-\lambda(A, 2)) A^{\omega}\left[L\left(A^{-1}\right)\right]^{-1} \leqq \ldots$. Then if $0<\mu(1) \leqq \mu(2)$ $\leqq \cdots \mu(k) \rightarrow \infty$ as $k \rightarrow \infty$ are the eigenvalues of $S_{F^{-}}$restricted to $F^{\wedge} \mathscr{M}^{\wedge}$ the perturbation theorem will yield $(M-\lambda(A, k)) A^{\omega}\left[L\left(A^{-1}\right)\right]^{-1}=\mu(k)+o(1)$.

In the remainder of this paper all limits are taken as $A \rightarrow \infty$ unless stated otherwise. 
8. Convergence of $\left(S_{A}^{\wedge}\right)^{1 / 2}$ to $\left(S^{\wedge}\right)^{1 / 2}$ and $\left(S_{A}^{\wedge}\right)^{1 / 2} F_{A}^{\wedge}$ to $\left(S^{\wedge}\right)^{1 / 2} F^{\wedge}$-Case II. As in $\$ 5, F_{A}^{\wedge}=F^{\wedge}$ for all $A$.

We now show that $\left(S^{\wedge}\right)^{1 / 2}$ is the closure of the strong limit of $\left(S_{A}^{-}\right)^{1 / 2}$ and that $\left(S^{\wedge}\right)^{1 / 2} F^{\wedge}$ is the closure of the strong limit of $\left(S_{A}^{\wedge}\right)^{1 / 2} F^{\wedge}$.

We first state a well-known and easily verified result about slowly oscillating functions as a lemma.

Lemma 8a. If $L(\xi)$ is slowly oscillating as $\xi \rightarrow 0^{+}$, positive, continuous on $0<\xi<\infty, L(\xi)=O(1)$ as $\xi \rightarrow \infty$ and bounded away from 0 as $\xi \rightarrow \infty$, then

$$
\lim L\left(\xi_{1}\right)\left[L\left(\xi_{2}\right)\right]^{-1}=1
$$

as $\xi_{1} \rightarrow 0, \xi_{2} \rightarrow 0$, where $\xi_{1}, \xi_{2}$ satisfy $0<a<\xi_{1} \xi_{2}^{-1}<b<\infty$; and for each $\epsilon>0$ there exists a constant $C(\epsilon)$ such that

$$
L\left(\xi_{1}\right)\left[L\left(\xi_{2}\right)\right]^{-1} \leqq C(\epsilon)\left[\left(\xi_{1} \xi_{2}^{-1}\right)^{\iota}+\left(\xi_{1} \xi_{2}^{-1}\right)^{-\epsilon}\right]
$$

for all $0<\xi_{1}, \xi_{2}<\infty$.

Next, an easy computation shows that $S_{A}^{\hat{}} f \cdot(t)=s_{A}(t) f(t)$, where

$$
s_{A}(t)=A^{\omega}\left[L\left(A^{-1}\right)\right]^{-1}\left(M-F\left(t A^{-1}\right)\right) .
$$

LEMMA $8 \mathrm{~b}$. Under the assumptions of $\$ 7$ on $F(\xi)$ we have

$$
\lim s_{A}(t)=t^{\omega}, \quad 0 \leqq t<\infty ;
$$

for any $\epsilon>0$ there exists a positive constant $M(\epsilon)$ such that for all $A>0$

$$
0 \leqq s_{A}(t) \leqq M(\epsilon)\left(t^{*}+t^{-\epsilon}\right) t^{\omega} ;
$$

and for any $m_{1}>0$, there exists $A_{0}>0$ and $t_{0}>0$ such that

$$
s_{A}(t) \geqq m_{1} \text { for } t>t_{0}, A>A_{0} .
$$

Proof. By assumption, $M-F(\xi)=\xi^{\epsilon} L(\xi) \epsilon(\xi)$, where $\epsilon(\xi)$ is bounded, $\epsilon(\xi) \rightarrow 1$ as $\xi \rightarrow 0^{+}$and $\epsilon(\xi)=O\left(\xi^{-\omega}\right)$ as $\xi \rightarrow \infty$, and thus

$$
s_{A}(t)=t^{\omega} L\left(t A^{-1}\right)\left[L\left(A^{-1}\right)\right]^{-1} \epsilon\left(t A^{-1}\right) \text {. }
$$

For any fixed $t, 0<t<\infty$, (1) of Lemma 8a immediately gives (3).

From (6) we see that there exists a constant $C>0$ such that

$$
s_{A}(t) \leqq C t^{\omega} L\left(t A^{-1}\right)\left[L\left(A^{-1}\right)\right]^{-1}
$$

for $0<t<\infty, A>0$, and using (2) of Lemma 8a we get (4).

Now, given $\epsilon>0$, there exists $a(\epsilon)>0$ such that for $0<t A^{-1}<a(\epsilon)$ and $A^{-1}<a(\epsilon)$ we have

$$
\epsilon\left(t A^{-1}\right) \geqq m_{2}, \quad L\left(t A^{-1}\right)\left[L\left(A^{-1}\right)\right]^{-1} \geqq\left(t^{t}+t^{-t}\right)^{-1} .
$$

Hence for all sufficiently large $A$ and $0<t A^{-1}<a(\epsilon)$,

$$
s_{A}(t) \geqq m_{2} t^{\omega}\left(t^{t}+t^{-t}\right)^{-1} .
$$


Choose $m_{3}>0$ so that $M-F(\xi)>m_{3}$ for $\xi \geqq a(\epsilon)$. Then

$$
s_{A}(t) \geqq m_{3} A^{\omega}\left[L\left(A^{-1}\right)\right]^{-1}
$$

for $t A^{-1} \geqq a(\epsilon)$, etc.

THEOREM 8c. $\left(S^{\wedge}\right)^{1 / 2}$ is the closure of the strong limit of $\left(S_{A}^{\wedge}\right)^{1 / 2}$.

Proof. Let $f \in \mathscr{D}\left(\left(S^{\wedge}\right)^{1 / 2}\right)$ and $\epsilon>0$ be given. We must find a

$$
g \in \mathscr{D}\left(\left(S^{\wedge}\right)^{1 / 2}\right)
$$

such that $\|g-f\|<\epsilon,\left\|\left(S^{\wedge}\right)^{1 / 2} g-\left(S^{\wedge}\right)^{1 / 2} f\right\|<\epsilon$ and $\left(S_{A}^{\hat{A}}\right)^{1 / 2} g \rightarrow\left(S^{\wedge}\right)^{1 / 2} g$.

Let $f_{\delta}(t)=e^{-\delta t} f(t)$. Then

$$
\left\|f-f_{\delta}\right\|^{2}=\int_{0}^{\infty}\left|1-e^{-\delta t}\right|^{2}|f(t)|^{2} d \mu(t)
$$

and hence by Lebesgue's theorem, for $\delta$ sufficiently small

$$
\left\|f-f_{\delta}\right\|^{2}<\epsilon
$$

We also have

$$
\left\|\left(S^{\wedge}\right)^{1 / 2}\left(f-f_{\delta}\right)\right\|^{2}=\int_{0}^{\infty}|f(t)|^{2}\left|1-e^{-\delta t}\right|^{2} t^{\omega} d \mu(t)
$$

and since $f \in \mathscr{D}\left(\left(S^{\wedge}\right)^{1 / 2}\right)$ we see that for $\delta$ sufficiently small

$$
\left\|\left(S^{\wedge}\right)^{1 / 2}\left(f-f_{\delta}\right)\right\|<\epsilon .
$$

Choose $\delta$ so that (7) and (8) are satisfied and set $g=f_{\delta}$. Then

$$
\left\|\left(S^{\wedge}\right)^{1 / 2} g-\left(S_{A}^{-}\right)^{1 / 2} g\right\|^{2}=\int_{0}^{\infty}|f(t)|^{2} e^{-2 \delta t}\left|t^{\omega / 2}-s_{A}(t)^{1 / 2}\right|^{2} d \mu(t)
$$

and using (4) of Lemma $8 \mathrm{~b}$ we see that

$$
\left|t^{\omega / 2}-s_{A}(t)^{1 / 2}\right|^{2} \leqq 2 t^{\omega}\left[1+M(\epsilon)\left(t^{\epsilon}+t^{-\epsilon}\right)\right] .
$$

Now taking $\epsilon<\omega$, and using (3) of Lemma $8 \mathrm{~b}$ in conjunction with Lebesgue's theorem we get that

$$
\left\|\left(S^{\wedge}\right)^{1 / 2} g-\left(S_{A}^{\wedge}\right)^{1 / 2} g\right\| \rightarrow 0 .
$$

Finally we must show that $\left(S^{\wedge}\right)^{1 / 2} F^{\wedge}$ is the closure of the strong limit of $\left(S_{A}\right)^{1 / 2} F^{-}$.

It is for this proof that we need $\Omega$ to be an interval. In Theorem $8 \mathrm{c}$ it is trivial that the approximating function $f_{\delta}$ is in $\mathscr{D}\left(\left(S^{-}\right)^{1 / 2}\right)$. Here, given $f \in \mathscr{D}\left(\left(S^{\wedge}\right)^{1 / 2} F^{\wedge}\right)$ we must find an approximating function in

$$
\mathscr{D}\left(\left(S^{\wedge}\right)^{1 / 2} F^{\wedge}\right)
$$

and it is not clear that for an arbitrary $\Omega$ this is possible. Widom [14] gives conditions on $\Omega$ so that the approximation is possible, but it seems that the 
most natural situation here is to take $\Omega=[0, a]$ and without loss of generality, $[0,1]$.

THEOREM $8 \mathrm{~d} .\left(S^{\wedge}\right)^{1 / 2} F^{\wedge}$ is the closure of the strong limit of $\left(S_{A}^{\wedge}\right)^{1 / 2} F^{\curvearrowleft}$.

Proof. Let $f^{\wedge} \in \mathscr{D}\left(\left(S^{\wedge}\right)^{1 / 2} F^{\wedge}\right)$. It is clearly sufficient to consider two cases: (i) $F^{\wedge} f^{\wedge}=f^{\wedge}$ and (ii) $F^{\wedge} f^{\wedge}=0$.

Case (i). Let $g_{\lambda}$ be an even, nonnegative, infinitely differentiable function defined on $-\infty<y<\infty$, vanishing off $[-\lambda, \lambda]$, and such that

$$
\int_{0}^{\infty} g_{\lambda}(y) d \mu(y)=1
$$

for $\lambda>0$ and

$$
\int_{\delta}^{\infty} g_{\lambda}(y) d \mu(y) \rightarrow 0
$$

as $\lambda \rightarrow 0$ for all $\delta>0$. Clearly

$$
\left|g_{\lambda}^{\hat{\lambda}}(t)\right| \leqq 1
$$

and

$$
g_{\lambda}^{\wedge}(t) \rightarrow 1
$$

as $\lambda \rightarrow 0$.

Let $\mathscr{W}$ be the set of all functions, $f^{-}(t)$, such that $F^{\wedge} f^{\wedge}=f^{-}$and

$$
f^{\wedge} \in \mathscr{D}\left(\left(S^{\wedge}\right)^{1 / 2} F^{\wedge}\right) \text {. }
$$

Then $F f=f$ and $f$ vanishes off $[0,1]$. Let $\mathscr{W}_{1} \subseteq \mathscr{W}$ be all functions $f^{\wedge} \in \mathscr{W}$ such that $f$ vanishes off $[0, \theta]$ for some $\theta, 0<\theta<1$.

Let $f^{\wedge} \in \mathscr{W}_{1}$ and set $f_{\lambda}^{\wedge}(t)=g_{\lambda}^{\wedge}(t) f^{\wedge}(t)$. Then

$$
f_{\lambda}(y)=g_{\lambda} * f \cdot(y)=\int_{0}^{\theta} \int_{0}^{\lambda} g_{\lambda}(z) f(x) D(z, y, x) d_{\mu}(z) d_{\mu}(x),
$$

and since $D(z, y, x)=0$ if $z+x>y$, for $\lambda$ sufficiently small $f_{\lambda}(y)$ vanishes off $[0,1]$ and $F^{\wedge} f_{\lambda}^{\wedge} \cdot(t)=f_{\lambda}^{\wedge}(t)$. Using (9), (10), and Lebesgue's theorem, we obtain

$$
\left\|f_{\lambda}^{\wedge}-f^{\wedge}\right\| \rightarrow 0
$$

as $\lambda \rightarrow 0$.

Since $g_{\lambda}(y)$ is infinitely differentiable, $g_{\lambda}^{\wedge}(t)=O\left(t^{-r}\right)$ as $t \rightarrow \infty$ for all $r$. This and (9) imply that $f_{\lambda}^{\wedge} \in \mathscr{D}\left(\left(S^{\wedge}\right)^{1 / 2} F^{\wedge}\right)$. Using (9), (10) and the fact that $\left.f^{\wedge} \in \mathscr{L}\right)\left(\left(S^{\wedge}\right)^{1 / 2} F^{\wedge}\right)$ in conjunction with Lebesgue's theorem we get

$$
\left\|\left(S^{\wedge}\right)^{1 / 2} F^{\wedge}\left(f_{\lambda}^{\wedge}-f^{\wedge}\right)\right\| \rightarrow 0
$$

as $\lambda \rightarrow 0$.

Since $g_{\lambda}^{\wedge}(t)=O\left(t^{-r}\right)$ as $t \rightarrow \infty$ for all $r$ and $f_{\lambda}^{\wedge} \in \mathscr{U}\left(\left(S^{\wedge}\right)^{1 / 2} F^{\wedge}\right)$ the same 
proof as in Theorem $8 \mathrm{c}$ shows that

$$
\left\|\left(S_{A}^{\wedge}\right)^{1 / 2} F^{\wedge} f_{\lambda}^{-}-\left(S^{\wedge}\right)^{1 / 2} F^{\wedge} f_{\lambda}^{\wedge}\right\| \rightarrow 0 .
$$

Therefore if $f^{\wedge} \in \mathscr{W}_{1}$, there exists an $f_{\lambda}^{\wedge} \in \mathscr{L}\left(\left(S^{\wedge}\right)^{1 / 2} F^{\wedge}\right)$ such that $\left\|f_{\lambda}^{-}-f^{\wedge}\right\|$ $<\epsilon,\left\|\left(S^{\wedge}\right)^{1 / 2} F^{\wedge}\left(f_{\lambda}^{\wedge}-f^{\wedge}\right)\right\|<\epsilon$ and $\left(S_{A}^{\wedge}\right)^{1 / 2} F^{\wedge} f_{\lambda}^{\wedge} \rightarrow\left(S^{\wedge}\right)^{1 / 2} F^{\wedge} f_{\lambda}^{\wedge}$.

Now let $f^{\wedge} \in \mathscr{W}$. Set $g_{\theta}(y)=f\left(y \theta^{-1}\right), 0<\theta<1$. Then $g_{\theta}(y)$ vanishes off $[0, \theta]$ and we have

$$
\hat{g}_{\theta}^{\hat{\imath}}(t)=\int_{0}^{\infty} f\left(y \theta^{-1}\right) J(y t) d_{\mu}(y)=\theta^{2 \nu+1} f^{-}(\theta t) .
$$

Thus $\left.g_{\theta}^{\wedge} \in \mathscr{C}\right)\left(\left(S^{\wedge}\right)^{1 / 2} F^{\wedge}\right)$. It is easy to see that $\left\|f-g_{\theta}\right\| \rightarrow 0$ as $\theta \rightarrow 1$ and hence $\left\|f^{\wedge}-g_{\theta}^{\hat{\theta}}\right\| \rightarrow 0$ as $\theta \rightarrow 1$. Similarly $\left\|\left(S^{\wedge}\right)^{1 / 2} F^{\wedge}\left(f^{\wedge}-g_{\theta}^{\wedge}\right)\right\| \rightarrow 0$ as $\theta \rightarrow 1$. Since $g_{\theta}^{\wedge} \in \mathscr{W}_{1}$, we see that if $f^{\wedge} \in \mathscr{W}$ there exists an $h^{\wedge} \in \mathscr{W}_{1}$ such that $\left\|f^{\wedge}-h^{\wedge}\right\|<\epsilon$ and $\left\|\left(S^{\wedge}\right)^{1 / 2} F^{\wedge}\left(f^{\wedge}-h^{\wedge}\right)\right\|<\epsilon$.

Thus we have the theorem for functions in $\mathscr{W}$. If $F^{\wedge} f^{\wedge}=0$ the proof is trivial.

9. The asymptotic formula-Case II. Let $S_{F^{-}}$be constructed from $F^{\wedge}$ and $S^{\wedge}$ as in Theorem $3 a$. We recall that $S_{F^{-}}$is a self-adjoint operator on $\mathscr{M}^{\wedge}$, the closure in $\mathscr{C}^{-}$of $\mathscr{Y}^{\wedge}$, where $\mathscr{Y}^{\wedge}=\left\{f \mid f \in \mathscr{C}^{\wedge}, F^{\wedge} f \in \mathscr{D}\left(\left(S^{-}\right)^{1 / 2}\right)\right\}$, and $\mathscr{D}\left(S_{F^{-}}\right) \subseteq \mathscr{F}^{\circ}$.

Because $\Omega=[0,1], \mathscr{H}^{\wedge}$ is dense in $\mathscr{C}^{-}$and $S_{F^{-}}$is a self-adjoint operator on $\mathscr{C}^{\wedge}$. Let

$$
\hat{S_{F^{-}}}=\int_{0^{-}}^{\infty} \lambda d \psi^{-}(\lambda)
$$

be the spectral resolution of $\hat{S_{F^{-}}}$on $\mathscr{C}^{-}$and let

$$
\hat{S_{A, F^{-}}}=\int_{0^{-}}^{\infty} \lambda d \hat{\psi_{A}}(\lambda)
$$

be the spectral resolution of $S_{A, F^{-}}=F^{\wedge} S_{A} \hat{F}{ }^{\wedge}$. Then using Theorems $8 \mathrm{c}$ and $8 \mathrm{~d}$ in conjunction with Theorem $3 \mathrm{~d}$ we have

$$
\psi_{\hat{A}}(\lambda) f \rightarrow \hat{\psi}(\lambda) f
$$

for all $f \in \mathscr{C}^{\wedge}$ and $0 \leqq \lambda<\infty, \lambda$ not in the point spectrum of $\hat{S_{F}}$. We define $R^{-}$as the restriction of $S_{F}^{-}$to $F^{\wedge} \mathscr{C}^{\wedge}$ and $R_{A}^{-}$as the restriction of $\hat{S}_{\hat{A}, F^{-}}$to $F^{\wedge} \mathscr{C}^{\wedge}$.

It is easy to see that $R^{\wedge}>0$ and $R_{A}^{\wedge}>0$. Thus $R^{\wedge}$ has the spectral resolution on $F^{\wedge} \mathscr{C}^{-}$

$$
R^{`}=\int_{0}^{\infty} \lambda d G^{`}(\lambda)
$$

where $G^{\wedge}(\lambda)=\psi^{\wedge}(\lambda)-\psi^{\wedge}(0)$ for $0<\lambda<\infty$, and $G^{\wedge}(0)=0$, and $R_{A}^{\wedge}$ has 
the spectral resolution on $F^{-1}{ }^{-}$

$$
R_{A}^{-}=\int_{0}^{\infty} \lambda d G_{A}^{\wedge}(\lambda)
$$

where $G_{\hat{A}}^{\hat{n}}(\lambda)=\psi_{\hat{A}}(\lambda)-\psi_{\hat{A}}(0)$ for $0<\lambda<\infty$, and $G_{\hat{A}}^{\hat{n}}(0)=0$. Since $\psi^{\wedge}(0)$ $=I-F^{\wedge}$ and $\psi_{A}(0)=I-F^{\wedge}$ it follows from (1) that

$$
\hat{G_{A}} \hat{(\lambda)} \rightarrow G(\lambda)
$$

for $0 \leqq \lambda<\infty$ and $\lambda$ not in the point spectrum of $R$. Here " $\rightarrow$ " is in $\mathscr{L}^{\prime}$.

Lemma 9a. Let $A(1)<A(2)<\cdots, A(k) \rightarrow \infty$ as $k \rightarrow \infty$. Let $f_{n} \in F^{\wedge} \mathscr{C}^{\wedge}$, $\left\|f_{n}\right\|=1$, and $\left(\hat{R_{A(n)}} f_{n} \mid f_{n}\right) \leqq m<\infty$ for $n \in p$. If $f_{n} \rightarrow f$ as $n \rightarrow \infty$ in $p_{1}$ a subsequence of $\boldsymbol{p}$, then $f \neq 0$.

Proof. By Lemma 6 a $f_{n}(t) \rightarrow f(t)$ uniformly on any compact set. By Lemma $8 \mathrm{~b}$ we have that given $m_{1}>0$, there exists $A_{0}>0$ and $t_{0}>0$ such that $s_{A}(t)$ $\geqq m_{1}$ for $t>t_{0}, A>A_{0}$. The rest of the proof is the same as that of Lemma $6 \mathrm{~b}$.

Theorem 9b. Let $F$ satisfy (i), (ii), (iii), of $\$ 7$ and $\Omega=[0,1]$. If $\lambda(A, 1)$ $\geqq \lambda(A, 2) \geqq \cdots$ are the positive eigenvalues of $B_{A}$ and $0<\mu(1) \leqq \mu(2) \leqq \cdots$, $\mu(k) \rightarrow \infty$ as $k \rightarrow \infty$ are the positive eigenvalues of $S_{F^{*}}$, then

$$
\lambda(A, k)=M-A^{-\omega} L\left(A^{-1}\right)(\mu(k)+o(1)) .
$$

Proof. Lemma 9a and (2) are the hypotheses of Theorem 3e. Hence $(A(n))^{\omega}\left[L\left((A(n))^{-1}\right)\right]^{-1}(M-\lambda(A(n), k))=\mu(k)+o(1)$ for $k=1,2, \cdots$ and this is equivalent to (3).

10. Definitions and preliminaries-Case III. We shall assume that $F$ satisfies the following conditions:

(i) $F$ is a bounded real-valued function in $L_{1, \mu}$;

(ii) $F$ has a unique maximum $M$ at $\xi_{0} \neq 0$ and $\lim \sup F<M$ as $\xi \rightarrow \infty$;

(iii) $M-F(\xi) \sim \sigma_{1}\left|\xi-\xi_{0}\right|^{\omega} L\left(\xi-\xi_{0}\right)$ as $\xi \rightarrow \xi_{0}^{+}, \quad M-F(\xi) \sim \sigma_{2}\left|\xi-\xi_{0}\right|^{\omega}$ $L\left(\xi-\xi_{0}\right)$ as $\xi \rightarrow \xi_{0}$, where $\omega>0, \sigma_{1}>0, \sigma_{2}>0$ and $L(\xi)$ is an even, positive, continuous function that is slowly oscillating as $\xi \rightarrow 0$ and is bounded and bounded away from zero as $\xi \rightarrow \infty$.

We also assume that $\Omega=[0,2 \pi]$.

We define four Hilbert spaces. Let $\otimes$ and $\mathscr{H}^{-}$both be $L_{2, \mu}$ and denote the norm by \|\|$_{\mu}$. Let $\mathscr{\psi}_{j}$ and $\mathcal{H}_{j}{ }^{-}$both be $L^{2}[(-\infty, \infty)]$ with respect to Lebesgue measure and denote the norm by \|\| .

We define the unitary maps $\phi$ of $\mathscr{E}$ onto $\mathscr{H}^{\prime}$ and $\psi$ of $\mathscr{C}$ onto $\mathscr{C}^{-}$by

$$
\phi f \cdot(\xi)=\int_{0}^{\infty} J(x \xi) f(x) d \mu(x)
$$

and 


$$
\psi f \cdot(t)=\int_{-\infty}^{\infty} e^{2 x i y t} f(y) d y
$$

where the partial integrals converge in the metric of $\mathscr{H}^{\wedge}$ and $\mathscr{C}^{\wedge}$, respectively.

Maps $\chi_{A}$ of $\mathscr{E}^{-}$to $\mathscr{C}^{-}$and $\chi_{A}^{*}$ of $\mathscr{C}^{-}$to $\mathscr{H}^{-}$are defined by

$$
\begin{aligned}
\chi_{A} f \cdot(t) & =f\left(\xi_{0}+t A^{-1}\right)\left(C_{\nu} A^{-1}\right)^{1 / 2}\left(\xi_{0}+t A^{-1}\right)^{\nu} & & \text { for } t>-\xi_{0} A, \\
& =0 & & \text { for } t \leqq-\xi_{0} A
\end{aligned}
$$

and

$$
\begin{aligned}
\chi_{A}^{*} g \cdot(\xi) & =g\left(A\left(\xi-\xi_{0}\right)\right)\left(C_{\nu} A^{-1}\right)^{-1 / 2} \xi^{-\nu} & & \text { for } \xi>0, \\
& =0 & & \text { for } \xi<0 .
\end{aligned}
$$

It is easy to see that $\chi_{A}$ is an isometric map into $\mathscr{C}^{-}$and that $\chi_{A}^{*}$ is a partially isometric map onto $\mathscr{H}^{-}$whose partial domain is the range of $\chi_{A}$. Thus $\chi_{A}^{*} \chi_{A}=I$ and $\chi_{A} \chi_{A}^{*}=I$ on the range of $\chi_{A}$.

The operators $E_{A}, E_{A}^{\hat{A}}, F_{\hat{A}}, T, T^{\wedge}$, and $T_{A}^{\hat{\alpha}}$ are defined as in $\$ 4$, using the maps $\phi, \psi, \chi_{A}$, and $\chi_{A}^{*}$ of this section. Let the operators $S_{A}{ }^{\wedge}$ and $S^{\wedge}$ be defined on $\mathscr{C}^{\wedge}$ by

$$
\hat{S_{A}} \hat{f} \cdot(t)=s_{A}(t) f(t)
$$

and

$$
S^{\wedge} f \cdot(t)=s(t) f(t)
$$

where

$$
\begin{aligned}
s_{A}(t) & =A^{\omega}\left[L\left(A^{-1}\right)\right]^{-1}\left[M-F\left(\xi_{0}+t A^{-1}\right)\right] & & \text { for } t>-\xi_{0} A, \\
& =0 & & \text { for } t<-\xi_{0} A
\end{aligned}
$$

and

$$
\begin{array}{rlr}
s(t) & =\sigma_{1}|t|^{\omega} & \text { for } t<0, \\
& =\sigma_{2}|t|^{\omega} \quad & \text { for } t<0 .
\end{array}
$$

We note that

$$
\hat{S_{A}} \hat{f} \cdot(t)=A^{\omega}\left[L\left(A^{-1}\right)\right]^{-1} \hat{T_{A}} \hat{f} \cdot(t) .
$$

Finally we define the projections $F$ in $\mathscr{C}$ and $F^{-}$in $\mathscr{C}^{-}$by

$$
\begin{aligned}
F f \cdot(y) & =f(y) & & \text { for }-1 \leqq y \leqq 1, \\
& =0 & & \text { for } y>1
\end{aligned}
$$

and

$$
F^{\wedge}=\psi F \psi^{-1}
$$


In this case, $f * g$ will denote convolution in $L^{1}(-\infty, \infty)$ with respect to Lebesgue measure.

If $\lambda(A, 1) \geqq \lambda(A, 2) \geqq \cdots$ are the positive eigenvalues of $B_{A}$, then $[M-\lambda(A, 1)] A^{\omega}\left[L\left(A^{-1}\right)\right]^{-1} \leqq[M-\lambda(A, 2)] A^{\omega}\left[L\left(A^{-1}\right)\right]^{-1} \leqq \cdots$ are the eigenvalues of $F_{A} \hat{S} S_{A} F_{A}$ restricted to $F_{A} \hat{C^{*}}$. As in the two previous cases we show that $F_{A} \hat{S_{A}} F_{A}$ converges to $S_{F^{-}}$of Theorem 3 a such that if $0<\mu(1)$ $\leqq \mu(2) \leqq \cdots$ are the eigenvalues of $S_{F^{-}}$restricted to $F^{\wedge} \mathscr{C}^{\circ}$ then

$$
[M-\lambda(A, k)] A^{\omega}\left[L\left(A^{-1}\right)\right]^{-1}=\mu(k)+o(1) .
$$

11. Convergence of $\left(S_{A}^{\wedge}\right)^{1 / 2}$ to $\left(S^{\wedge}\right)^{1 / 2}$ and $F_{A}^{\wedge}$ to $F^{-}$-Case III.

Lemma 11a. With the definitions of $\$ 10$ we have

(1) $\lim s_{A}(t)=s(t)$;

(2) for any $\epsilon>0$, there is a constant $M(\epsilon)$ independent of $t,-\infty<t<\infty$ and $A>0$ such that

$$
s_{A}(t) \leqq M(\epsilon)|t|^{\omega}\left[|t|^{\epsilon}+|t|^{-\epsilon}\right]
$$

(3) given $m_{1}>0$ there are numbers $A_{0}>0, t_{0}>0$ such that for $A>A_{0}$ and $|t|>t_{0}, s_{A}(t) \geqq m_{1}$.

Proof. The proof is virtually the same as that of Lemma $8 \mathrm{~b}$.

THEOREM $11 \mathrm{~b} .\left(S^{\wedge}\right)^{1 / 2}$ is the closure of the strong limit of $\left(S_{A}\right)^{1 / 2}$.

Proof. Let $f \in \mathscr{D}\left(\left(S^{\wedge}\right)^{1 / 2}\right)$ and $\epsilon>0$ be given. We must find a

$$
g \in \mathscr{D}\left(\left(S^{\wedge}\right)^{1 / 2}\right)
$$

such that $\|f-g\|<\epsilon,\left\|\left(S^{\wedge}\right)^{1 / 2}(f-g)\right\|<\epsilon$ and $\left(S_{A}^{\wedge}\right)^{1 / 2} g \rightarrow\left(S^{\wedge}\right)^{1 / 2} g$. If $f_{\delta}(t)=e^{-\delta t} f(t)$ then just as in Theorem $8 \mathrm{c}, g=f_{\delta}$ works if $\delta$ is sufficiently small.

Theorem 11c. If $F_{A^{\wedge}}$ and $F^{\wedge}$ are defined as in $\$ 10$, then $F^{\wedge}$ is the strong limit of $\hat{F_{A}}$ as $A \rightarrow \infty$.

Proof. Let

$$
\begin{aligned}
& P(u, w, t, A) \\
& \quad=J_{\nu-1 / 2}\left(u\left(A \xi_{0}+t\right)\right) J_{\nu-1 / 2}\left(u\left(A \xi_{0}+w\right)\right)\left(u\left(A \xi_{0}+t\right)\right)^{1 / 2}\left(u\left(A \xi_{0}+w\right)\right)^{1 / 2} .
\end{aligned}
$$

A straightforward computation shows that

$$
\begin{aligned}
F_{A}^{\hat{g}} \cdot(t) & =\int_{0}^{2 \pi} \int_{-\xi_{0} A}^{\infty} P(u, w, t, A) g(w) d w d u & & \text { for } t>-A \xi_{0}, \\
& =0 & & \text { for } t<-A \xi_{0} .
\end{aligned}
$$

In what follows $C$ is a generic constant. 
Let $\mathscr{W}^{-}$be the set of functions $f \in \mathscr{C}^{\wedge}$ which are continuous and have support in $|t| \leqq a$ for some $a$. We first prove that if $g \in \mathscr{W}^{\wedge}$, then $\boldsymbol{F}_{A} \boldsymbol{g} \cdot(t)$ $\rightarrow F^{\wedge} g \cdot(t)$ uniformly on $[-b, b]$ for any $b<\infty$.

For $A$ sufficiently large and $|t| \leqq b$

$$
F_{A} g \cdot(t)=\int_{0}^{2 \pi} \int_{-a}^{a} P(u, w, t, A) g(w) d w d u
$$

and we can write $\hat{F_{A}} g \cdot(t)=I_{1}+I_{2}$, where

$$
\begin{aligned}
& I_{1}=\int_{0}^{\delta} \int_{-a}^{a} P(u, w, t, A) g(w) d w d u, \\
& I_{2}=\int_{0}^{2 \pi} \int_{-a}^{a} P(u, w, t, A) g(w) d w d u .
\end{aligned}
$$

Now using (8) 82 and the well-known fact that $x^{\nu} J_{\nu}(x)=O(1)$ as $x \rightarrow 0$ we see that

$$
J_{\nu-1 / 2}\left(u\left(A \xi_{0}+t\right)\right)\left[u\left(A \xi_{0}+t\right)\right]^{1 / 2} \leqq C
$$

and hence

$$
\left|\int_{-a}^{a} P(u, w, t, A) g(w) d w\right|<C
$$

for $0 \leqq u \leqq 2 \pi, A>0$, and $t>-\xi_{0} A$. Thus $I_{1} \leqq C \delta$ for $|t| \leqq b$.

We will show in a moment that

$$
\lim \int_{-a}^{a} P(u, w, t, A) g(w) d w=\int_{-a}^{a} g(w) \cos (u(t-w)) d w
$$

uniformly for $|t|<b$. Using this and Lebesgue's limit theorem, see (6), we will have

$$
\lim I_{2}=\int_{\delta}^{2 \pi} \int_{-a}^{a} g(w) \cos (u(t-w)) d w d u .
$$

From (8) $§ 2$ and a standard trigonometric identity,

$$
P(u, w, t, A)=\pi^{-1}\left\{\cos (u(t-w))+\cos \left(u\left(2 A \xi_{0}+t+w\right)-\nu \pi\right)\right\}+O\left(A^{-1}\right),
$$

where the $O\left(A^{-1}\right)$ is uniform for $\delta \leqq u \leqq 2 \pi,|t| \leqq b,|w| \leqq a$. Thus

$$
\begin{aligned}
& \lim \int_{-a}^{a} P(u, w, t, A) g(w) d w=\pi^{-1}\left\{\int_{-a}^{a} g(w) \cos (u(t-w)) d w\right. \\
& \left.\quad+\lim \int_{-a}^{a} \cos \left(u\left(2 A \xi_{0}+t+w\right)-\nu \pi\right) g(w) d w+\lim \int_{-a}^{a} O\left(A^{-1}\right) g(w) d w\right\} .
\end{aligned}
$$

The second limit on the right is zero by the Riemann-Lebesgue theorem; the third integral is obviously zero, and it is easy to check that convergence is 
uniform for $\delta \leqq u \leqq 2 \pi,|t| \leqq b$. Therefore we have (7).

Let

$$
I_{3}=\pi^{-1} \int_{0}^{2 \pi} \int_{-a}^{a} g(w) \cos (u(t-w)) d w d u .
$$

Then clearly $\hat{F_{A}} \dot{g} \cdot(t) \rightarrow I_{3}$ uniformly as $A \rightarrow \infty$ for $|t| \leqq b$.

Set $\Lambda=[-2 \pi, 2 \pi]$. Then using the fact that $C_{\Lambda}(u)$ is even we get

$$
I_{3}=\int_{-\infty}^{\infty} C_{\Lambda}(2 \pi u) e^{i 2 \pi u t} \int_{-\infty}^{\infty} g(w) e^{-i 2 \pi u u} d w d u .
$$

But since $C_{1}(2 \pi u)$ is the characteristic function of $[-1,1],(9)$ is just $I_{3}=\psi F \psi^{-1} g \cdot(t)=F^{\wedge} g \cdot(t)$ and we have proved our assertion.

We now show that if $f \in \mathscr{W}^{-}$, then

$$
F_{A}^{\wedge} f \rightarrow F^{\wedge} f \text {. }
$$

Indeed, if $g \in \mathscr{C}^{-}$, we get

$$
\left|\left(\left[F_{A}^{\hat{f}} f-F^{-} f\right] \mid g\right)\right|^{2} \leqq 4\|g\|^{2}\|f\|^{2}
$$

using Schwarz's inequality and the fact that since $F_{A}^{\wedge}$ and $F^{\wedge}$ are projections, $\left\|F^{\wedge}\right\|=1$ and $\left\|F_{A}\right\|=1$ for all $A$. Hence there is a $C>0$ such that

$$
\left|\int_{|t| \geqq C}\left[F_{A} \hat{f} f \cdot(t)-F^{\wedge} f \cdot(t)\right](g(t))^{*} d t\right| \leqq \epsilon / 2
$$

for all $A$. But by what we have just proved

$$
\int_{|t| \leqq C}\left[F_{A}^{\wedge} f \cdot(t)-F^{\wedge} f \cdot(t)\right](g(t))^{*} d t \rightarrow 0 .
$$

Now (10) follows trivially from (11) and (12).

Since $\mathscr{W}^{\wedge}$ is dense in $\mathscr{C}^{\wedge}$, again using the fact that $\left\|F^{\wedge}\right\|=1$ and $\left\|F_{A}^{\wedge}\right\|=1$ we see that $F_{A}^{\hat{f}} f-F^{\wedge} f$ for all $f \in \mathscr{C}^{\wedge}$. But weak convergence of projections implies strong convergence and our theorem is proved.

12. Convergence of $\left(S_{A}^{\wedge}\right)^{1 / 2} F_{A}$ to $\left(S^{\wedge}\right)^{1 / 2} F^{-}$-Case III. Consider the rectangle $R$ shown in Figure 1.

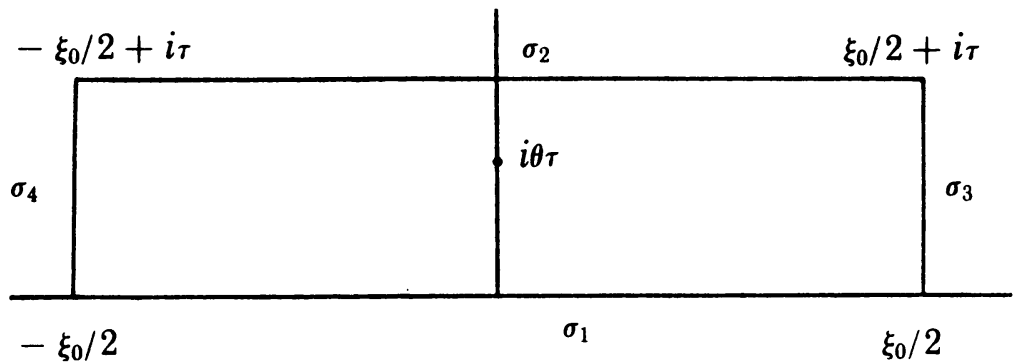


Let $\gamma_{k}(i \theta \tau)$ be the harmonic measure of the side $\sigma_{k}$ at the point $i \theta \tau, k=1,2$, 3,4. That is, $\gamma_{k}(z)$ is harmonic on $R$ and $\gamma_{k}(z)=1$ on the interior of $\sigma_{k}$ and $\gamma_{k}(z)=0$ on $\sigma_{j}, j \neq k$.

LEMMA 12a. With the above notations we have the inequalities

(i) $\gamma_{1}(i \tau \theta) \geqq 1-\theta-2 \theta \tau \xi_{0}^{-1} \cosh \pi \tau \xi_{0}^{-1}$,

(ii) $\gamma_{2}(i \tau \theta)<\theta$,

(iii) $\gamma_{3}(i \tau \theta)<\tau \theta \xi_{0}^{-1} \cosh \pi \tau \xi_{0}^{-1}$,

(iv) $\gamma_{4}(i \tau \theta)<\tau \theta \xi_{0}^{-1} \cosh \pi \tau \xi_{0}^{-1}$.

Proof. The demonstration of this result follows routine lines. See [3] where it is given in detail.

A straightforward computation shows that

$$
\begin{aligned}
F_{A} g \cdot(t) & =\int_{0}^{2 \pi} Q(u, t, A) a(u, A) d u & & \text { for } t>-A \xi_{0}, \\
& =0 & & \text { for } t \leqq-A \xi_{0},
\end{aligned}
$$

where

$$
\begin{aligned}
a(u, A) & =\int_{-\xi_{0} A}^{\infty} C_{\nu} J\left(u\left(A \xi_{0}+w\right)\right)\left[A \xi_{0}+w\right]^{\nu} g(w) d w, \\
Q(u, t, A) & =J_{\nu-1 / 2}\left[u\left(A \xi_{0}+t\right)\right]\left(A \xi_{0}+t\right)^{1 / 2} u^{\nu+1 / 2} .
\end{aligned}
$$

Let $\mathscr{D}$ be the set of functions $h(y)$ in $\mathscr{C}$ which are infinitely differentiable and have compact support and let $\mathscr{D}^{-}=\psi \mathscr{D}$. Let $\mathscr{D}_{1}$ be the subset of $\mathscr{D}$ consisting of those functions that have support on $|y| \leqq C$ for some $C<1$ and let $\mathscr{D}_{1}{ }^{\wedge}=\psi \mathscr{D}_{1}$. Let $\mathscr{D}_{2}$ be the subset of $\mathscr{D}$ consisting of those functions that have support on $|y| \geqq C$ for some $C>1$, let $\mathscr{D}_{2}{ }^{\wedge}=\psi \mathscr{D}_{2}$.

Theorem 12b. If $g \in \mathscr{D}_{1}{ }^{\circ}$ or $g \in \mathscr{D}_{2}{ }^{\wedge}$ and if $a(u, A)$ is defined by (2) then

$$
\Delta^{N} a(2 \pi, A)=O\left(A^{-r}\right)
$$

and

$$
\frac{d}{d u} \Delta^{N} a(2 \pi, A)=O\left(A^{-r}\right)
$$

as $A \rightarrow \infty$ for all $r$ and $N=0,1,2, \cdots$, where

$$
\Delta=\left(\frac{d}{d u}\right)^{2}+\frac{2 \nu}{u} \frac{d}{d u}
$$

Proof. Let $g \in \mathscr{D}_{1}^{-}$. Using (5) $\S 2$ we see that

$$
\Delta^{N} a(u, A)=(-1)^{N} \int_{-\xi_{0} A}^{\infty} C_{\nu} J\left[u\left(A \xi_{0}+w\right)\right]\left(A \xi_{0}+w\right)^{\nu+2 N} g(w) d w .
$$

We write 


$$
\Delta^{N} a(u, A)=(-1)^{N}\left(a_{1}+a_{2}+a_{3}\right),
$$

where

$$
\begin{aligned}
& a_{1}=\int_{-\xi_{0} A}^{-\delta_{1} A} C_{\nu} J\left(u\left(A \xi_{0}+w\right)\right)\left[A \xi_{0}+w\right]^{\nu+2 N} g(w) d w, \\
& a_{2}=\int_{-\delta_{1} A}^{\delta_{2} A} C_{\nu} J\left[u\left(A \xi_{0}+w\right)\right]\left(A \xi_{0}+w\right)^{\nu+2 N} g(w) d w, \\
& a_{3}=\int_{\delta_{2} A}^{\infty} C_{\nu} J\left[u\left(A \xi_{0}+w\right)\right]\left(A \xi_{0}+w\right)^{\nu+2 N} g(w) d w .
\end{aligned}
$$

Here $-\xi_{0} A<-\delta_{1} A<0<\delta_{2} A<\infty$ and $\delta_{1}, \delta_{2}>0$. $\delta_{1}$ and $\delta_{2}$ will be chosen precisely later.

It is well known that if $g \in \mathscr{D}^{-}$then $g(t)=O\left(t^{-r}\right)$ as $t \rightarrow \infty$ for all $r$. Using this and (2) $\$ 2$ we easily obtain that $\left|a_{1}\right|=O\left(A^{-r}\right)$ and $\left|a_{3}\right|=O\left(A^{-r}\right)$ as $A \rightarrow \infty$ for all $r$.

From the standard relation

$$
J_{\nu-1 / 2}=\frac{1}{2}\left[H_{\nu-1 / 2}^{(1)}(z)+H_{\nu-1 / 2}^{(2)}(z)\right]
$$

it follows that $a_{2}=a_{2}^{+}+a_{2}^{-}$, where

$$
a_{2}^{+}=\frac{1}{2} u^{1 / 2-\nu} \int_{-\delta_{1} A}^{\delta_{2} A} H_{\nu-1 / 2}^{(1)}\left(u\left[A \xi_{0}+w\right]\right)\left(A \xi_{0}+w\right)^{2 N+1 / 2} g(w) d w
$$

and

$$
a_{2}^{-}=\frac{1}{2} u^{1 / 2-\nu} \int_{-\delta_{1} A}^{\delta_{2} A} H_{\nu-1 / 2}^{(2)}\left(u\left[A \xi_{0}+w\right]\right)^{2 N+1 / 2} g(w) d w .
$$

Applying Cauchy's theorem to the integrand of $a_{2}^{+}$with respect to the top curve of Figure 2 and to the integrand of $a_{2}^{-}$with respect to the bottom

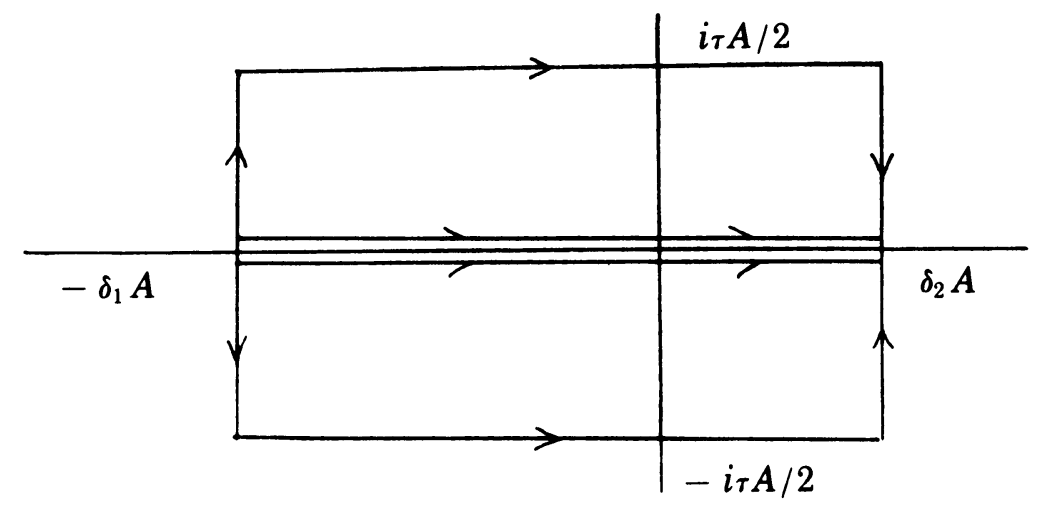

Figure 2 
curve we see that $a_{2}^{+}=I_{1}^{+}+I_{2}^{+}+I_{3}^{+}$and $a_{2}^{-}=I_{1}^{-}+I_{2}^{-}+I_{3}^{-}$, where

$$
\begin{array}{ll}
I_{1}^{+}=\int_{-\delta_{1} A}^{-\delta_{1} A+i \tau A / 2}, \quad I_{2}^{+}=\int_{-\delta_{1} A+i_{\tau} A / 2}^{\delta_{2} A+i_{\tau} A / 2}, \quad I_{3}^{+}=\int_{\delta_{2} A+i_{\tau} A / 2}^{\delta_{2} A}, \\
I_{1}^{-}=\int_{-\delta_{1} A}^{-\delta_{1} A-i \tau A / 2}, \quad I_{2}^{-}=\int_{-\delta_{1} A-i \tau A / 2}^{\delta_{2} A-i \tau A / 2}, \quad I_{3}^{-}=\int_{\delta_{2} A-i_{\tau} A / 2}^{\delta_{2} A},
\end{array}
$$

the integrand of $I_{j}^{+}$being that of $a_{2}^{+}$and the integrand of $I_{j}^{-}$being that of $a_{2}^{-}$

We consider $I_{2}^{+}$first. It is easily seen that $g \in \mathscr{D}_{1}^{-}$implies $g\left(v+i_{\tau} A / 2\right)$ $=O\left(e^{C_{\pi}+A}\right)$ as $A \rightarrow \infty$. Using the estimate (6) $\$ 2$ for $H_{\nu-1 / 2}^{(1)}$, we see that for $u=2 \pi$ the integrand of $I_{2}^{+}$is $O\left(A^{2 N} e^{\pi(C-1) \tau A}\right)$ as $A \rightarrow \infty$ and as $C<1$ we see that $I_{2}^{+}=O\left(A^{-r}\right)$ as $A \rightarrow \infty$ for all $r . I_{2}^{-}$is handled in exactly the same way using estimate (7) $\$ 2$ for $H_{\nu-1 / 2}^{(2)}$.

We next examine $I_{1}^{+}$. Consider the rectangle $R$ of Figure 3 .

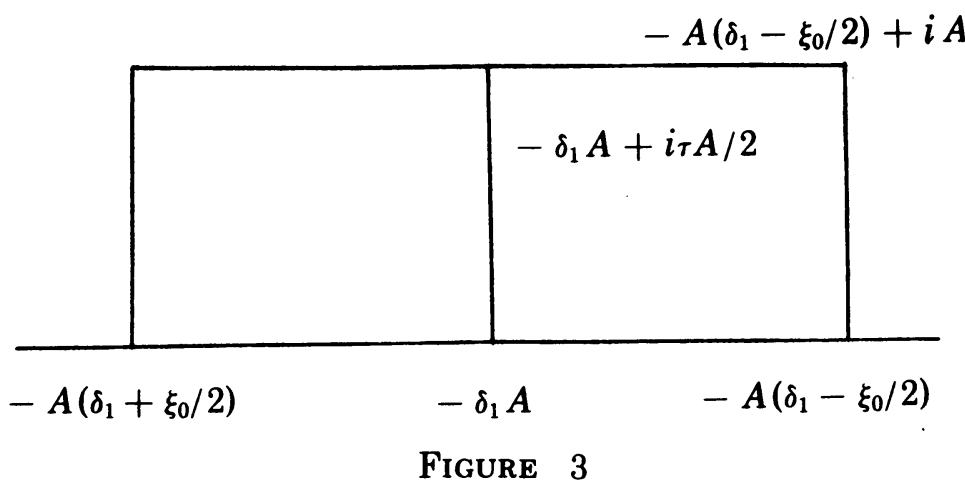

Choose $\delta_{i}$ to satisfy $\xi_{0} / 2<\delta_{i}<\xi_{0}, i=1,2$. Then $|g(w)| \leqq C_{1} A^{-r}$ on the bottom side of $R$. On the other three sides we have $|g(w)| \leqq C_{1} e^{2 \pi r C A}$. Throughout this argument $C_{1}$ will be a generic constant.

Now $R$ is conformally equivalent to the rectangle of Lemma $12 \mathrm{a}$. Using the notation there and one of the standard principles of harmonic measure we get

$$
\begin{aligned}
\log \mid g\left(-\delta_{1} A+\right. & i A \tau \theta) \mid \leqq \gamma_{1}(i \tau \theta) \log \left(C_{1} A^{-r}\right) \\
& +\left[\gamma_{2}(i \tau \theta)+\gamma_{3}(i \tau \theta)+\gamma_{4}(i \tau \theta)\right] \log \left(C_{1} e^{2 \pi \tau C A}\right)
\end{aligned}
$$

Using the estimates of Lemma $12 \mathrm{a}$ we can choose $\tau$ so small that $\gamma_{1}(i \tau \theta) \geqq 1 / 3$ and $\gamma_{2}(i \tau \theta)+\gamma_{3}(i \tau \theta)+\gamma_{4}(i \tau \theta)<\theta$ for $0 \leqq \theta \leqq 1 / 2$. Hence

$$
\left|g\left(-\delta_{1} A+i A \eta\right)\right| \leqq C_{1} A^{-r / 3} e^{2 \pi A C_{\eta}}
$$

for $0 \leqq \eta \leqq \tau / 2$. Using the estimate (6) $\S 2$

$$
\left(A \xi_{0}+w\right)^{2 N+1 / 2} H_{\nu-1 / 2}^{(1)}\left(2 \pi\left(A \xi_{0}+w\right)\right) \leqq C_{1} e^{-2 \pi A n} A^{2 N}
$$

for $0 \leqq \eta \leqq \tau / 2$, where $w=-\delta_{1} A+i \eta A$. Therefore the integrand of $I_{1}^{+}$is 
$O\left(A^{2 N-r / 3} e^{-2 \pi(1-C) \eta A}\right)$ as $A \rightarrow \infty$ for $0 \leqq \eta \leqq \tau / 2$. Since $1-C>0$ and $r$ is arbitrary, $I_{1}^{+}=O\left(A^{-r}\right)$ for all $r$ as $A \rightarrow \infty$.

$I_{3}^{+}, I_{1}^{-}$, and $I_{2}^{-}$are handled in the same way. Thus we have proved (4) if $g \in \mathscr{D}_{1}^{i}$.

Now let $g \in \mathscr{D}_{2}^{i}$. Then $\psi^{-1} g \cdot(y)$ has its support on $1<C \leqq|y| \leqq C_{0}$ $<\infty$ and $g(w)=g_{1}(w)+g_{2}(w)$, where

$$
g_{1}(w)=\int_{C}^{C_{0}} \psi^{-1} g \cdot(y) e^{i 2 x y w} d y, \quad g_{2}(w)=\int_{C}^{C_{0}} \psi^{-1} g \cdot(y) e^{i 2 \pi y w} d y .
$$

Then $a_{2}=a_{21}+a_{22}$, where

$$
\begin{aligned}
& a_{21}=\int_{-\delta_{1} A}^{\delta_{2} A} J_{\nu-1 / 2}\left(u\left(A \xi_{0}+w\right)\right)\left[A \xi_{0}+w\right]^{2 N+1 / 2} u^{1 / 2-\nu} g_{1}(w) d w, \\
& a_{22}=\int_{-\delta_{1} A}^{\delta_{2} A} J_{\nu-1 / 2}\left(u\left(A \xi_{0}+w\right)\right)\left[A \xi_{0}+w\right]^{2 N+1 / 2} u^{1 / 2-\nu} g_{2}(w) d w .
\end{aligned}
$$

Applying Cauchy's theorem as in the previous case we obtain $a_{21}=I_{11}$ $+I_{21}+I_{31}$ and $a_{22}=I_{12}+I_{22}+I_{32}$, where

$$
\begin{array}{lll}
I_{11}=\int_{-\delta_{1} A}^{-\delta_{1} A+i A_{\tau} / 2}, & I_{21}=\int_{-\delta_{1} A+i A_{\tau} / 2}^{\delta_{2} A+i A_{\tau} / 2}, & I_{31}=\int_{\delta_{2} A-i A_{\tau} / 2}^{\delta_{2} A}, \\
I_{12}=\int_{-\delta_{1} A}^{-\delta_{1} A-i A_{\tau} / 2}, I_{22}=\int_{-\delta_{1} A-i A_{\tau} / 2}^{\delta_{2} A-i A_{\tau} / 2}, & I_{32}=\int_{\delta_{2} A-i A_{\tau} / 2}^{\delta_{2} A} .
\end{array}
$$

The proof from this point is so similar to the previous case that we omit it.

Finally we consider the proof of (5). By (4) $\$ 2$ we have that

$$
\begin{aligned}
& \frac{d}{d u} \Delta^{N} a(u, A) \\
& \quad=(-1)^{N+1} \int_{-\xi_{0} A}^{\infty} u^{1 / 2-v} J_{v+1 / 2}\left[u\left(A \xi_{0}+w\right)\right]\left(A \xi_{0}+w\right)^{2 N+3 / 2} g(w) d w .
\end{aligned}
$$

But this is the same problem as (4) and of course has exactly the same solution.

Lemma 12c. Let $g \in \mathscr{D}_{1}^{\wedge}$ or $g \in \mathscr{D}_{2}^{\wedge}$. Then for every non-negative integer $N$ and $c>0$

$$
\lim \sup \int_{-A \xi_{0}}^{c A}|t|^{2 N}\left|F_{A} g \cdot(t)\right|^{2} d t \leqq 2^{2 N} \int_{-\infty}^{\infty} t^{2 N}\left(2+t^{2}\right)^{N}|g(t)|^{2} d t .
$$

Proof. Let $g \in \mathscr{D}_{1}^{\wedge}$. Set $h(t, A)=2 A \xi_{0} t\left(1+t / 2 A \xi_{0}\right)$ and

$$
g_{N, A}(t)=(h(t, A))^{N} g(t) .
$$

Let $\Delta$ be as in Theorem $12 \mathrm{~b}$ and set $\Delta_{A}=\Delta+\left(A \xi_{0}\right)^{2}$. Finally let $a(u, A)$ be as in Theorem $12 \mathrm{~b}$. We first show that 


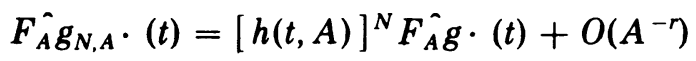

for all $r$ and $-A \xi_{0} \leqq t \leqq c A$.

We note the following evident facts:

$$
\Delta^{N} a(u, A)=O(1), \quad \frac{d}{d u} \Delta^{N} a(u, A)=O(u)
$$

as $u \rightarrow 0$. Also an easy computation using (4) $\$ 2$ and (5) $\$ 2$ shows that

$$
\frac{d}{d u} u^{2 \nu} \frac{d}{d u} J\left(u\left(A \xi_{0}+t\right)\right)=u^{2 \nu} \Delta J\left(u\left(A \xi_{0}+t\right)\right) .
$$

Now, since

$$
\left(-\Delta_{A}\right)^{N} J\left(u\left(A \xi_{0}+w\right)\right)=[h(w, A)]^{N} J\left(u\left(A \xi_{0}+w\right)\right)
$$

we see that

$$
\hat{F_{A} g_{N, A}} \cdot(t)=C_{\nu} \int_{0}^{2 \pi} J\left(u\left(A \xi_{0}+t\right)\right)\left(A \xi_{0}+t\right)^{\nu} u^{2 \nu}\left(-\Delta_{A}\right)^{N} a(u, A) d u .
$$

Performing $2 N$ integrations by parts and making repeated use of (8) and (9) we obtain

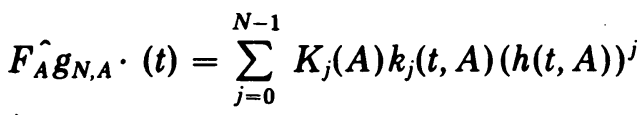

$$
+\left(A \xi_{\sigma}+t\right) \sum_{j=0}^{N-1} P_{j}(A) p_{j}(t, A)(h(t, A))^{j}+(h(t, A))^{N} F_{A} g \cdot(t) .
$$

The terms $K_{j}(A)$ and $P_{j}(A)$ arise from the terms $\left(-\Delta_{A}\right)^{k} a(2 \pi, A)$ and

$$
\frac{d}{d u}\left(-\Delta_{A}\right)^{k} a(2 \pi, A)
$$

respectively, and by Theorem $12 \mathrm{~b}$ are $O\left(A^{-r}\right)$ as $A \rightarrow \infty$ for all $r$. The terms $k_{j}(t, A)$ and $p_{j}(t, A)$ arise from the terms $\left(A \xi_{0}+t\right)^{\nu} J\left(2 \pi\left(A \xi_{0}+t\right)\right)$ and $\left(A \xi_{0}+t\right)^{\nu} J^{\prime}\left[2 \pi\left(A \xi_{0}+t\right)\right]$, respectively, and are thus uniformly bounded for all $t$ and $A$. Thus we have proved (7). Now

$$
h(t, A) \geqq A \xi_{0} t
$$

for $t>-A \xi_{0}$ and

$$
(h(t, A))^{2} \leqq\left(2 A \xi_{0} t\right)^{2}\left(2+t^{2}\right)
$$

for $-\infty<t<\infty$ and $A>2^{-1 / 2} \xi_{0}$. Using (7), (12) and the fact that $\left\|F_{\hat{A}} \hat{\|}\right\|=1$ we get

$$
\int_{-A \xi_{0}}^{c A}|h(t, A)|^{2 N}\left|F_{A} g \cdot(t)\right|^{2} d t \leqq\left(2 A \xi_{0}\right)^{2 N} \int_{-\infty}^{\infty} t^{2 N}\left(2+t^{2}\right)^{N}|g(t)|^{2} d t+O\left(A^{-r}\right)
$$


for $A>2^{-1 / 2} \xi_{0}$. Now using (11) and dividing through by $\left(A \xi_{0}\right)^{2 N}$ we have for $A$ sufficiently large and all $r$

$$
\int_{-A \xi 0}^{c A} t^{2 N}\left|\hat{F_{A} g} \cdot(t)\right|^{2} d t \leqq 2^{2 N} \int_{-\infty}^{\infty} t^{2 N}\left(2+t^{2}\right)^{N}|g(t)|^{2} d t+O\left(A^{-r}\right) .
$$

The lemma clearly follows.

Theorem $12 \mathrm{~d}$. If $g \in D_{1}^{\wedge}$ then

$$
\lim \left\|\left(S^{\wedge}\right)^{1 / 2} F^{\wedge} g-\left(S_{A}\right)^{1 / 2} F_{A}^{\wedge} g\right\|=0 .
$$

Proof. First we set

$$
\left\|\left(S^{\wedge}\right)^{1 / 2} F^{\wedge} g-\left(S_{A}\right)^{1 / 2} F_{A}^{\wedge} g\right\|^{2}=I_{1}+I_{2},
$$

where

$$
\begin{aligned}
& I_{1}=\int_{-\infty}^{0}\left|\left(\sigma_{2} t^{\omega}\right)^{1 / 2} F^{\wedge} g-\left(s_{A}(t)\right)^{1 / 2} F_{A}^{\wedge} g\right|^{2} d t, \\
& I_{2}=\int_{0}^{\infty}\left|\left(\sigma_{1} t^{\omega}\right)^{1 / 2} F^{\wedge} g-\left(s_{A}(t)\right)^{1 / 2} F_{A}^{\wedge} g\right|^{2} d t .
\end{aligned}
$$

Now $I_{1}=I_{11}+I_{12}+I_{13}$, where

$$
\begin{aligned}
I_{11} & =\int_{-\infty}^{-A \xi_{0}} \sigma_{2}|t|^{\omega}\left|F^{\wedge} g\right|^{2} d t, \\
I_{12} & =\int_{-A \xi_{0}}^{-T}\left|\left(\sigma_{2}|t|^{\omega}\right)^{1 / 2} F^{\wedge} g-\left(s_{A}(t)\right)^{1 / 2} F_{A}^{\wedge} g\right|^{2} d t, \\
I_{13} & =\int_{-T}^{0}\left|\left(\sigma_{2}|t|^{\omega}\right)^{1 / 2} F^{-} g-\left(s_{A}(t)\right)^{1 / 2} F_{A} g\right|^{2} d t,
\end{aligned}
$$

and $I_{2}=I_{21}+I_{22}+I_{23}$, where

$$
\begin{aligned}
& I_{21}=\int_{\xi_{0} A}^{\infty}\left|\left(\sigma_{1}|t|^{\omega}\right)^{1 / 2} F^{\wedge} g-\left(s_{A}(t)\right)^{1 / 2} F_{A} g\right|^{2} d t, \\
& I_{22}=\int_{T}^{\xi_{0} A}\left|\left(\sigma_{1}|t|^{\omega}\right)^{1 / 2} F^{\wedge} g-\left(s_{A}(t)\right)^{1 / 2} F_{A}^{\wedge} g\right|^{2} d t, \\
& I_{23}=\int_{0}^{T}\left|\left(\sigma_{1}|t|^{\omega}\right)^{1 / 2} F^{\wedge} g-\left(s_{A}(t)\right)^{1 / 2} F_{A} g\right|^{2} d t .
\end{aligned}
$$

Clearly $\lim I_{11}=0$. Next

$$
I_{12} \leqq 2\left(K_{1}+K_{2}\right),
$$

where

$$
K_{1}=\int_{-\xi_{0}}^{-T} \sigma_{2}|t|^{\omega}|g(t)|^{2} d t
$$


and

$$
K_{2}=\int_{-A_{\xi_{0}}}^{-T} s_{A}(t)\left|\hat{F_{A}} \bar{g} \cdot(t)\right|^{2} d t .
$$

It is evident that for $T$ sufficiently large and all $A$ we have $K_{1} \leqq \epsilon / 8$. Now choose $N$ so that $\omega+1=2 N-a$ where $a>0$. Then using (2) $\$ 11$ and Lemma $12 \mathrm{c}$ we have

$$
\lim \sup K_{2} \leqq C 2^{2 N} T^{-a} \int_{-\infty}^{\infty} t^{2 N}\left(2+t^{2}\right)^{N}|g(t)|^{2} d t .
$$

Thus for $T$ sufficiently large lim $\sup K_{2}<\epsilon / 8$ and

$$
\lim \sup I_{12} \leqq \epsilon / 2 .
$$

In the same way, for $T$ sufficiently large

$$
\lim \sup I_{22} \leqq \epsilon / 2 .
$$

Fix $T$ so that (13) and (14) are satisfied. Since $s_{A}(t) \rightarrow s(t)$ boundedly for $-T \leqq t \leqq T$ and $F_{A} g \rightarrow F^{\wedge} g$ in $\mathscr{C}^{\wedge}$ we have

$$
\lim \left(I_{13}+I_{23}\right)=0 .
$$

Finally $I_{21} \leqq 2\left(K_{1}+K_{2}\right)$, where here

$$
K_{1}=\int_{5_{0} A}^{\infty} \sigma_{1}|t|^{\omega}|g(t)|^{2} d t
$$

and

$$
K_{2}=\int_{\xi_{0} A}^{\infty} s_{A}(t)\left|F_{A} g \cdot(t)\right|^{2} d t
$$

Clearly

$$
\lim K_{1}=0 .
$$

Recalling the definition of $s_{A}(t),(1) \S 10$, we see that

$$
s_{A}(t) \leqq C A^{\omega+1}
$$

for $A$ sufficiently large and all $t$. Now if $t \geqq \xi_{0} A$, then $h(A, t) \leqq 3 t^{2}$ and in this case (10) of Lemma 12c reduces to

$$
F_{A} g_{N, A} \cdot(t)=O\left(A^{-r}\right) O\left(t^{2 N-1}\right)+[h(t, A)]^{N} F_{A} g \cdot(t)
$$

for all $r$. We also note that for $t \geqq 0$

$$
h(A, t) \geqq t^{2} .
$$

From (18) we get that 


$$
\left|F_{\hat{A}} g \cdot(t)\right|^{2} \leqq 2[h(t, A)]^{-2 N}\left\{\left|F_{\hat{A}} g_{N, A} \cdot(t)\right|^{2}+O\left(A^{-r}\right) O\left(t^{4 N-2}\right)\right\} .
$$

Now applying (12), (17), (19) and the fact that $\left\|F_{\hat{A}}\right\|=1$, we obtain for sufficiently large $A$ and all $r$

$$
K_{2} \leqq C A^{\omega+1-2 N} \int_{-\infty}^{\infty} t^{2 N}\left(2+t^{2}\right)^{N}|g(t)|^{2} d t+O\left(A^{-r}\right) .
$$

Choosing $N$ so that $2 N>\omega+1$, we see that

$$
\lim K_{2}=0 \text {. }
$$

Combining (13), (14), (15), (16) and (20) we finally get

$$
\lim \sup \left\|\left(S^{\wedge}\right)^{1 / 2} F^{\wedge} g-\left(S_{A}^{\hat{A}}\right)^{1 / 2} F_{\hat{A}} g\right\|<\epsilon
$$

and as $\epsilon$ is arbitrary the theorem is proved.

We remark that in the analogous theorems of Hirschman and Widom, the integral $I_{21}$ does not appear.

Theorem 12e. Let $g \in \mathscr{D}_{2}$. Then

$$
\lim \left\|\left(S^{\wedge}\right)^{1 / 2} F^{\wedge} g-\left(\hat{S_{A}}\right)^{1 / 2} F_{A}^{\hat{A}} g\right\|=0 .
$$

Proof. The proof is the same as that of Theorem 12d. Note that in this case $F^{\wedge} g=0$.

THEOREM 12f. $\left(S^{\wedge}\right)^{1 / 2} F^{\wedge}$ is the closure of the strong limit of $\left(S_{A}^{\wedge}\right)^{1 / 2} F_{A}$.

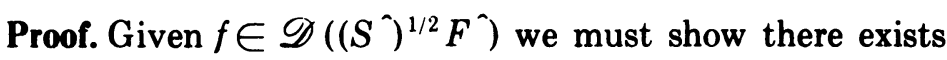

$$
h \in \mathscr{D}\left(\left(S^{\wedge}\right)^{1 / 2} F^{\wedge}\right)
$$

such that

(i) $\|f-h\|<\epsilon,\left\|\left(S^{\wedge}\right)^{1 / 2} F^{\wedge}(f-h)\right\|<\epsilon$,

(ii) $\left(S_{A}^{-}\right)^{1 / 2} F_{A} h \rightarrow\left(S^{\wedge}\right)^{1 / 2} F^{\wedge} h$.

It is sufficient to consider two cases. $F^{\wedge} f=f$ and $F^{\wedge} f=0$.

Suppose that $F^{\wedge} f=f$. For $0<\theta<1$, set $g_{\theta}(t)=f(\theta t)$. Then $\psi^{-1} g_{\theta} \cdot(y)$ $=\theta^{-1} \psi^{-1} f \cdot\left(y \theta^{-1}\right)$. Now $F^{\wedge} f=f$ implies $\psi^{-1} f \cdot(y)^{\wedge}=0$ for $|y|>1$ and hence $\psi^{-1} g_{\theta} \cdot(y)=0$ for $|y|>\theta$. Clearly $\psi^{-1} g_{\theta} \in \mathscr{D}\left(\left(S^{-}\right)^{1 / 2} F^{\wedge}\right)$. It is also evident that for $\theta$ sufficiently near 1 ,

$$
\left\|f-g_{\theta}\right\|<\epsilon / 2, \quad\left\|\left(S^{\wedge}\right)^{1 / 2} F^{\wedge}\left(f-g_{\theta}\right)\right\|<\epsilon / 2 .
$$

For each $\lambda>0$, let $h_{\lambda}(y)$ be a non-negative, even, infinitely-differentiable function that vanishes for $|y| \geqq \lambda$ and $\int_{-\infty}^{\infty} h_{\lambda}(y) d y=1$.

Then $\left|\psi h_{\lambda} \cdot(t)\right| \leqq 1, \psi h_{\lambda} \cdot(t) \rightarrow 1$ as $\lambda \rightarrow 0$ for all $t$, and $\psi h_{\lambda} \cdot(t)=O\left(t^{-r}\right)$ as $t \rightarrow \infty$ for all $r$ and fixed $\lambda$. Using this information and Lebesgue's theorem we get 


$$
\left\|\left(\psi h_{\lambda}\right) g_{\theta}-g_{\theta}\right\|<\epsilon / 2
$$

for $\lambda$ sufficiently small. Next we note that $\psi^{-1} g_{\theta} * h_{\lambda} \cdot(y)=0$ for $|y|>\lambda+\theta$. If $\lambda$ is such that

$$
\lambda+\theta<1
$$

then $\left(\psi h_{\lambda}\right) g_{\theta} \in \mathscr{D}_{\hat{1}}^{\hat{0}}$. Using $\left|\psi h_{\lambda} \cdot(t)\right| \leqq 1, g_{\theta}(t) \psi h_{\lambda} \cdot(t) \rightarrow g_{\theta}(t)$ as $\lambda \rightarrow 0$, and $g_{\theta} \in \mathscr{D}\left(\left(S^{\wedge}\right)^{1 / 2} F^{\wedge}\right)$ we see that for $\lambda$ sufficiently small

$$
\left\|\left(S^{\wedge}\right)^{1 / 2} F^{\wedge}\left(g_{\theta}\left(\psi h_{\lambda}\right)-g_{\theta}\right)\right\|<\epsilon / 2 .
$$

Choose $\theta$ so that (21) is satisfied and then choose $\lambda$ so that (22), (23), and (24) are satisfied. Set $h=\left(\psi h_{\lambda}\right) g_{\theta}$. Then (i) is fulfilled. It is evident that $h \in \mathscr{D}\left(\left(S^{\wedge}\right)^{1 / 2} F^{\wedge}\right)$ and since $h \in \mathscr{D}_{1}^{\wedge}$ by Theorem $12 \mathrm{~d}$ we have (ii).

Suppose now that $F^{\wedge} f=0$. Then $\psi^{-1} f^{\wedge}(y)=0$ for $|y|<1$. Choose $1<C_{1}<C_{2}<\infty$ such that if $g(y)=\psi^{-1} f \cdot(y)$ for $C_{1}<|y|<C_{2}$ and $g(y)=0$ otherwise, then $\left\|g-\psi^{-1} f\right\|<\epsilon / 2$. Clearly $F^{\wedge} \psi g=0$ and $\|\psi g-f\|$ $<\epsilon / 2$. Now set $h=\left(\psi h_{\lambda}\right)(\psi g)$, where $\lambda$ is chosen so small that $C_{1}-\lambda>1$ and $\|h-\psi g\|<\epsilon / 2$. Then $h \in \mathscr{D}_{2}{ }^{\circ},\left\|\left(S^{\wedge}\right)^{1 / 2} F^{\wedge}(f-h)\right\|=0$, and $\|f-h\|$ $<\epsilon$, and the result follows from Theorem $12 \mathrm{e}$.

13. The asymptotic formula-Case III. Let $S_{F^{-}}$be constructed from $F^{\wedge}$ and $S^{\wedge}$ as in Theorem $3 a$. Then as in Case II, $S_{F^{-}}$is a self-adjoint operator on $\mathscr{C}^{-}$.

Let

$$
\hat{S_{F^{-}}}=\int_{0}^{\infty} \lambda d \psi^{\wedge}(\lambda)
$$

be the spectral resolution of $S_{F^{-}}^{-}$on $\mathscr{C}^{-}$and let

$$
\hat{S_{A, F^{-}}}=\int_{0}^{\infty} \lambda d \psi_{\hat{A}}(\lambda)
$$

be the spectral resolution of $\hat{S_{A, F^{-}}}=\hat{F_{A}} \widehat{S_{A}} \hat{F_{A}}$. Using Theorems $12 \mathrm{e}, 11 \mathrm{c}$, and $11 \mathrm{~b}$ in conjunction with Theorem $3 \mathrm{~d}$ we have

$$
\psi_{A}(\lambda) \rightarrow \psi^{\wedge}(\lambda)
$$

for every $\lambda$ not in the point spectrum of $S_{F^{-}}, 0 \leqq \lambda<\infty$. Now define $R^{\wedge}$ as $S_{F^{-}}$restricted to $F^{\wedge} \mathscr{C}^{\wedge}=\mathscr{N}^{\wedge}$ and let $R_{A}^{\hat{A}}$ be $S_{\hat{A}, F^{-}}$restricted to $F_{A}^{\wedge} \mathscr{C}^{-}$ $=\mathscr{N}_{A}^{\wedge}$. As in the previous cases $R^{\wedge}>0$ and $R_{A}^{\wedge}>0$ and we have the spectral resolutions

$$
R^{\wedge}=\int_{0}^{\infty} \lambda d G^{\wedge}(\lambda)
$$

on $\mathscr{N}^{\wedge}$, where $G^{\wedge}(\lambda)=\psi^{\wedge}(\lambda)-\psi^{\wedge}(0)$ and $G^{\wedge}(0)=0$ and 


$$
R_{A}^{\hat{A}}=\int_{0}^{\infty} \lambda d G_{\hat{A}}^{\hat{(}(\lambda)}
$$

on $\mathscr{N}_{\hat{A}}^{\hat{\lambda}}$, where $\hat{G_{A}}(\lambda)=\psi_{\hat{A}}(\lambda)-\psi_{\hat{A}}(0)$ and $G_{\hat{A}}^{\hat{A}}(0)=0$. Since $\psi^{\wedge}(0)=I$ $-F^{\wedge}$ and $\psi_{\hat{A}}(0)=I-F_{\hat{A}}$ it follows from (1) and Theorem $11 \mathrm{c}$ that

$$
G_{A}^{\hat{A}}(\lambda) \rightarrow G^{\wedge}(\lambda)
$$

for $0 \leqq \lambda<\infty$ and $\lambda$ not in the point spectrum of $R^{-}$.

LEMma 13a. Let $A(1)<A(2)<\cdots, A(n) \rightarrow \infty$ as $n \rightarrow \infty$. With the above definitions let $f_{n} \in \mathscr{N}_{A(n)}^{(},\left\|f_{n}\right\|=1$ and $\left(R_{A(n)} f_{n} \mid f_{n}\right) \leqq m<\infty$ for $n \in p$, where $p$ is a subsequence of $1,2,3, \ldots$. If $f_{n} \rightarrow f$ as $n \rightarrow \infty$ in $p_{1}, a$ subsequence of $p$, then $f \neq 0$.

Proof. Let

$$
f^{\#}(y)=\int_{0}^{\infty} f(x) J_{v-1 / 2}(y x)(y x)^{1 / 2} d x .
$$

It is well known, see Titchmarsh $[9$, p. 473], that

$$
\int_{0}^{\infty}\left|f^{\#}(y)\right|^{2} d y=\int_{0}^{\infty}|f(x)|^{2} d x
$$

Noting that $f_{n} \in \mathscr{N}_{A(n)}$ implies $f_{n}(t)=\hat{F_{A(n)}} f_{n} \cdot(t)$ a substitution gives

$$
f_{n}(t)=C_{\nu}^{2} \int_{0}^{2 \pi}\left(\left(A(n) \xi_{0}+t\right) u\right)^{n} J\left(u\left(A(n) \xi_{0}+t\right)\right)
$$

$$
\left.\times \int_{-A \xi_{0}}^{\infty}\left(\left(A(n) \xi_{0}+w\right) u\right)^{\nu} J\left(u\left(A(n) \xi_{0}+w\right)\right) f_{n}(w)\right) d w d u
$$

for $t>-A(n) \xi_{0}$ and zero otherwise. Setting $f_{A, n}(w)=f_{n}\left(w-A(n) \xi_{0}\right)$ we write (3) as

$$
\begin{aligned}
f_{n}(t) & =\left(C_{\Omega} f_{A, n}^{\#}\right)^{\#} \cdot\left(t+A(n) \xi_{0}\right) & & \text { for } t>-A(n) \xi_{0}, \\
& =0 & & \text { for } t \leqq-A(n) \xi_{0} .
\end{aligned}
$$

Then

$$
\left\|f_{n}\right\|^{2}=\int_{0}^{\infty}\left|\left(C_{\Omega} f_{A, n}^{*}\right)^{\#} \cdot(t)\right|^{2} d t=\int_{0}^{2 \pi}\left|f_{A, n}(w)\right|^{2} d w=1 .
$$

Now using the Schwarz inequality on (3) in conjunction with (4) and the fact that $\left(A(n) \xi_{0}+t\right) u J\left(u\left(A(n) \xi_{0}+t\right)\right) \leqq C$ for $0 \leqq u \leqq 1, t>-A(n) \xi_{0}$, and $n$ in $p_{1}$ we obtain

$$
\left|f_{n}(t)\right|^{2} \leqq C^{2}
$$

Now, by (4) $\$ 2$ we get 


$$
\begin{aligned}
\frac{d}{d t}\left\{\left(\left(A \xi_{0}+t\right) u\right)^{\nu} J\left(u\left(A \xi_{0}+t\right)\right)\right\} & \\
=\left(A \xi_{0}+t\right)^{-1}\left\{\nu J_{v-1 / 2}\right. & \left(u\left(A \xi_{0}+t\right)\right)\left[u\left(A \xi_{0}+t\right)\right]^{1 / 2} \\
& \left.-u^{2} J_{v+1 / 2}\left(u\left(A \xi_{0}+t\right)\right)\left[u\left(A \xi_{0}+t\right)\right]^{-(v+1 / 2)}\right\}
\end{aligned}
$$

and thus differentiating $f_{n}(t)$ we see that for $t \geqq-A(n) \xi_{0}$

$$
\left|f_{n}^{\prime}(t)\right| \leqq C\left(A(n) \xi_{0}+t\right)^{-1}
$$

Hence, given $t_{0}$, there is an $n_{0}$ such that for $n>n_{0}$ and $|t|<t_{0}$ (6)

$$
\left|f_{n}^{\prime}(t)\right| \leqq C \text {. }
$$

Now (5) and (6) imply that $f_{n}, n$ in $p_{1}$, are uniformly bounded and equicontinuous on any interval $|t| \leqq t_{0}$. Therefore since $f_{n} \rightarrow f$ as $n \rightarrow \infty$ in $p_{1}$ we have (if $f$ is suitably redefined on a set of measure zero),

$$
f_{n}(t) \rightarrow f(t)
$$

uniformly for $|t| \leqq t_{0}$.

By Lemma 11a, given $m_{1}>0$ there exist $A_{0}>0$ and $t_{0}>0$ such that for $A>A_{0},|t|>t_{0}$

$$
s_{A}(t) \geqq m_{1} .
$$

Taking $m_{1}>m$ and $n>n_{0}$, where $A\left(n_{0}\right)>A_{0}$, we have

$$
m \geqq m_{1} \int_{|t|>\omega_{0}}\left|f_{n}(t)\right|^{2} d t
$$

Hence

$$
\int_{|t| \leqq t_{0}}\left|f_{n}(t)\right|^{2} d t \geqq 1-m m_{1}^{-1}>0
$$

and by (7)

$$
\int_{|t| \leqq \iota_{0}}|f(t)|^{2} d t>0 .
$$

That is, $f \neq 0$.

Theorem 13b. Let $F$ satisfy (i), (ii), and (iii) of $\$ 10$ and $\Omega=[0,2 \pi]$. If $\lambda(A, 1) \geqq \lambda(A, 2) \geqq \cdots$ are the positive eigenvalues of $B_{A}$ and if $0<\mu(1)$ $\leqq \mu(2) \leqq \cdots, \mu(k) \rightarrow \infty$ as $k \rightarrow \infty$, are the positive eigenvalues of $S_{F^{-}}$then

$$
\lambda(A, k)=M-L\left(A^{-1}\right) A^{\omega}(\mu(k)+o(1))
$$

as $A \rightarrow \infty$ for each fixed $k=1,2, \ldots$.

Proof. Lemma 13a and (2) in conjunction with Theorem $3 e$ yield

$$
(A(n))^{\omega}\left[L\left((A(n))^{-1}\right)\right]^{-1}(M-\lambda(A(n), k))=\mu(k)+o(1)
$$


as $n \rightarrow \infty$ for $k=1,2, \ldots$. The theorem follows immediately.

\section{BIBLIOGRAPHY}

1. A. Erdélyi et al., Higher transcedental functions, Vol. II, McGraw-Hill, New York, 1953.

2. I. I. Hirschman, Jr., Variation diminishing Hankel transforms, J. Analyse Math. 8(1960/61), 307-336.

3. __ Extreme eigenualues of Toeplitz forms associated with Jacobi polynomials, Pacific J. Math. 14(1964), 107-161.

4. M. Kac, W. L. Murdock, and G. Szegö, On the eigen-values of certain Hermitian forms, J. Rational Mech. Anal. 2(1953), 767-800.

5. S. V. Parter, Extreme eigenualues of Toeplitz forms and applications to elliptic difference equations, Trans. Amer. Math. Soc. 99(1961), 153-192.

6. 67(1961), 191-196.

7. , On the extreme eigenualues of Toeplitz matrices, Trans. Amer. Math. Soc. 100(1961), $263-276$.

8. F. Riesz and B. Sz-Nagy, Leçons d'analyse fonctionnelle, 3ième éd., Gauthier-Villars, Paris, 1955.

9. E. C. Titchmarsh, Hankel transforms, Proc. London Math. Soc. 45(1922), 458-474.

10. G. N. Watson, $A$ treatise on the theory of Bessel functions, 2nd ed., Cambridge, 1962.

11. H. Widom, On the eigenualues of certain Hermitian operators, Trans. Amer. Math. Soc. 88(1958), 491-522.

12. Stable processes and integral equations, Trans. Amer. Math. Soc. 98(1961), 430-439.

13. , Extreme eigenualues of translation kernels, Trans. Amer. Math. Soc. 100(1961), 252-262.

14. Extreme eigenualues of $\mathrm{N}$-dimensional convolution operators, Trans. Amer. Math. Soc. 106(1963), 391-414.

UNIVERSITY OF CALIFORNIA, Berkeley, California 